\title{
Wilsonian renormalization group analysis of nonrelativistic three-body systems without introducing dimerons
}

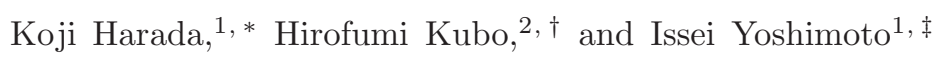 \\ ${ }^{1}$ Department of Physics, Kyushu University \\ Fukuoka 812-8581 Japan \\ ${ }^{2}$ Synchrotron Light Application Center, Saga University \\ 1 Honjo, Saga 840-8502, Japan
}

(Dated: August 30, 2018)

\begin{abstract}
Low-energy effective field theory describing a nonrelativistic three-body system is analyzed in the Wilsonian renormalization group ( $R G$ ) method. No effective auxiliary field (dimeron) that corresponds to two-body propagation is introduced.

The Efimov effect is expected in the case of an infinite two-body scattering length, and is believed to be related to the limit cycle behavior in the three-body renormalization group equations (RGEs). If the one-loop property of the RGEs for the nonrelativistic system without the dimeron field, which is essential in deriving RGEs in the two-body sector, persists in the three-body sector, it appears to prevent the emergence of limit cycle behavior. We explain how the multi-loop diagrams contribute in the three-body sector without contradicting the one-loop property of the RGEs, and derive the correct RGEs, which lead to the limit cycle behavior. The Efimov parameter, $s_{0}$, is obtained within a few percent error in the leading orders. We also remark on the correct use of the dimeron formulation.

We find rich RG-flow structure in the three-body sector. In particular, a novel nontrivial fixed point of the three-body couplings is found when the two-body interactions are absent. We also find, on the two-body nontrivial fixed point, the limit cycle is realized as a loop of finite size in the space of three-body coupling constants when terms with derivatives are included.
\end{abstract}

\section{INTRODUCTION}

Nonrelativistic three-body systems have many interesting features. There have been long-standing discrepancies and anomalies between theoretical calculations and the experiments for several physical quantities in the three nucleon systems. (See Ref. [1] for a review.) Socalled Borromean nuclei, three-body bound states, any two of whose constituents do not form a bound state, are known to exist. (See Ref. [2] for a review.) V. Efimov showed that there are infinitely many three-body bound states with the ratios of the binding energies of the subsequent bound states being a universal constant, $E_{n+1} / E_{n} \equiv e^{-2 \pi / s_{0}}=1 / 515.03 \cdots$, when the two-body scattering length is infinite [3, 4]. This "Efimov effect" has recently been attracting much attention because of the advancing experimental control over the very cold atomic systems by using Feshbach resonance.

At low energies, the internal degrees of freedom of the particles (i.e., nucleons, nuclei and atoms) become irrelevant and the system is well described by an effective field theory (EFT), in which only the particles without internal structure interact locally. EFT is an efficient, modelindependent approach and admits a systematic improvement of the description.

Bedaque, Hammer, and van Kolck [5] first noticed that

\footnotetext{
*Electronic address: harada@phys.kyushu-u.ac.jp

$\dagger$ Electronic address: kubo@higgs.phys.kyushu-u.ac.jp

‡Electronic address: yoshimoto@higgs.phys.kyushu-u.ac.jp
}

the Efimov effect is related to the RG limit cycle behavior of the three-body nonderivative coupling of the EFT. $\mathrm{RG}$ analysis has played an important role in recognizing that the Efimov effect is a new kind of universal phenomena in the three-body systems [ 6 ]. There are several papers which deal with the Efimov effect in the light of nonperturbative RG analysis. For a recent review, see Ref. [7].

In their analysis, as well as subsequent studies by other authors, the so-called dimeron field [8], an auxiliary effective field which represents two-body propagation, is exploited. To our best knowledge, there is no literature in which the connection between the limit cycle behavior and the Efimov effect is established without a dimeron field. One might wonder if the limit cycle behavior can be obtained without introducing a dimeron field.

Although dimeron is a useful device, its introduction needs some care. It is difficult to establish the relations between a set of the coupling constants in the theory with dimeron and that in the original theory because of a nontrivial factor in the path-integral measure arising from the field transformation, an awkward contribution which depends the regularization of how to define a functional determinant. (In Appendix $\mathrm{A}$ we explain how the additional contribution from the Jacobian affects the relations among the couplings.) It is thus easier to think the theory with dimeron as another EFT, the couplings of which are to be determined by a matching procedure, than to make a direct connection between the two theories.

In this paper, we perform an RG analysis for a nonrelativistic three-body system of a single (complex) scalar 
field without introducing the dimeron. (It is straightforward to extend our analysis to the fermionic case.) The RGEs are derived and the limit cycle behavior of the three-body coupling is identified for the first time in this formulation. We also find that the usual formulation with dimeron field misses a certain contribution, which seems essential for obtaining the Efimov parameter $s_{0}$ within a few percent error.

It is well-known that nonperturbative RGE has the "one-loop" property, i.e., the running of the coupling constants is determined by a sum of one-loop diagrams with the propagator being the full one. In a nonrelativistic system, because of the particle number conservation and the absence of the antiparticle, there is no dressing for the propagator. This nonrelativistic feature divides the space of states into sectors labeled by particle numbers, and makes the RGEs in the two-body sector very simple: one only needs to consider actual one-loop diagrams that satisfy the particle number conservation, with the free propagator.

If this one-loop property (with free propagator) of the RGEs persists in the three-body sector, there appears to be no chance for the RGEs to have a limit cycle solution. This is a puzzling situation: on one hand, the Efimov effect implies the limit cycle behavior when the two-body couplings are tuned to the critical values, on the other hand, the general consideration given above implies the one-loop property, which seems to prevent the limit cycle behavior. It is one of our main results to explain how the one-loop property of the RGEs is consistent with contributions from multi-loop diagrams in the three-body sector. The resulting RGEs do allow limit cycle behavior.

We find very rich structures in the RG flow of the three-body coupling constants. In the leading-order calculation, in which only the nonderivative three-body coupling is included, we find a nontrivial fixed point on the two-body trivial fixed point. It implies the existence of a three-body strong coupling phase with the phase boundary on which the nontrivial fixed point resides. Note that such a nontrivial fixed point would only be revealed in the formulation without dimerons.

Extending the space of three-body operators up to including the terms with two derivatives, we find that, on the two-body nontrivial fixed point, the limit cycle is realized as a loop of finite size, and a nontrivial fixed point associated to it.

We also emphasize that the nonzero values of the twobody effective range and the off-shell parameter, which does not affect the on-shell two-body amplitude, modify the ratios of the binding energies of the three-body bound states. Although the effects of the nonzero value of the two-body effective range have been investigated [9, 10], those of the off-shell parameter have never been considered.

Several comments on the literature are in order. Although Bedaque, Hammer, and van Kolck [5] first showed the connection between the RG limit cycle and the Efi- mov effect, it does not come as a direct consequence of the RGE analysis, but rather from the scale-invariant behavior of the three-body amplitude. Actually they obtained the cutoff dependence of the coupling of the three-body nonderivative contact interaction after determining the Efimov parameter. Moroz, Floerchinger, Schmidt, and Wetterich [11] derived the Efimov parameter in the functional $\mathrm{RG}$ formulation with the dimeron field. Their leading-order value of the Efimov parameter contains about $40 \%$ errors. Their numerical calculation for the full RGE shows, however, that the resulting value of the Efimov parameter is in good agreement with the Efimov's.

There are several other papers devoted to the RG analysis of the three-body systems. For example, Diehl, Krahl and Schrerer 12] study the ratio of dimeronparticle scattering length of to that of particle-particle. Krippa, Walet, and Birse [13] do a similar thing for the ratio of dimeron-dimeron scattering length to that of particle-particle. All of these employ dimerons in their analysis.

The paper is organized as follows. In Sec. II we recapitulate the importance of Wilsonian RG analysis in revealing the physical features of few-body systems described by EFT. The one-loop property puzzle is explained and the solution is given in Sec. III] Several examples are shown for the illustration. We derive the RGEs for the coupling constant for the three-body interaction in the leading order in derivative expansion in Sec. IV. Comments on the difference between the dimeron formulation and ours are also given. In Sec. D we first concentrate on the case in which the two-body effective range and the off-shell parameter are zero. The RG flows are given and the Efimov parameter is obtained on the two-body nontrivial fixed point. The other cases are also examined. A novel nontrivial fixed point in the threebody coupling is identified. We examined the next-toleading order corrections in Sec. VI. The RG flow in the three-body coupling space is presented. The limit cycle is found to be realized as a loop of finite size in this space. We summarize the results in Sec. VII.

In Appendix A we illustrate a systematic way of introducing the dimeron field in the path integral formulation. We argue that there is a nontrivial Jacobian in the measure due to the nonlinear change of variables. It leads to change of coupling constants which has been neglected in the literature. In Appendix B, we demonstrate the derivation of the RGEs by using an example. In Appendix $\mathrm{C}$ we identify the shell-mode contribution of a two-loop diagram which is not obvious. In Appendix D. we explain that the pole appearing in the RGE for the three-body coupling is due to the existence of the twobody bound state. 


\section{EFFECTIVE FIELD THEORY IN THE THREE-BODY SECTOR AND WILSONIAN RG}

In this section, we recapitulate the basic idea of EFT [14] and the usefulness of Wilsonian RG [15] in the context of EFT.

\section{A. EFT}

Field theoretical description of nature has a hierarchical structure: at every scale there are relevant degrees of freedom and the interactions among them are described in terms of local operators subject to the symmetry of the system. Even though the system is composed of composite particles, if the momentum scale in question is smaller than the scale of the internal structure, one may neglect the structure and describe the system in terms of the fields corresponding to the particles. The effects of heavier particles that are not included in the EFT are encoded in the values of the coupling constants of local operators. Such a field theory is called an effective filed theory (EFT). A physical system may be described by various EFTs at various momentum scales.

The EFT description is very general: an EFT is defined by the relevant degrees of freedom, dimensions of spacetime, and the symmetries. It contains all possible operators with these attributes, which are infinitely many. A single EFT may describe several different systems that share the same attributes. Features of a specific system are reflected in the values of the coupling constants. Thus the EFT description is model independent.

The accuracy of the EFT description is controlled by power counting rules. Power counting rules tell us the degrees of importance ("orders") of operators. The higher the order is, the less important the contributions are. To a given order, one only needs to consider a finite number of operators and a restricted set of diagrams. If the power counting rules are consistent, counterterms are also supplied to the order. Thus the EFT description is systematically improvable.

\section{B. Wilsonian RG reveals nonperturbative aspects of EFT}

In several cases, nonperturbative physics shows up in the EFT context. It is of central importance to establish the power counting rules for such a case to extract physical information out of EFT. An example is the nuclear effective field theory (NEFT) 16-18] for the two-nucleon system in the $\mathrm{S}$ waves. The existence of a bound state, deuteron, in the spin-triplet channel, is a clear sign of nonperturbative dynamics.

It is the Wilsonian, or nonperturbative, RG analysis [15, 19 22] that reveals properties of the nonperturbative dynamics. The effects of quantum fluctuations are examined scale by scale by changing the cutoff in the
Wilsonian RG analysis, without relying on the perturbative approximation.

The application of such analyses to the NEFT in twonucleon sector is given in Refs. [23 25]. The nonperturbative feature of the system is translated into the RG language as the existence of a nontrivial fixed point. Because of the large anomalous dimensions, the power counting rules are modified from the perturbative ones based on the naive dimensional analysis.

\section{Efimov effect in the light of RG}

More than forty years ago, V. Efimov considered the case where the two-body scattering length is much larger than the range of the two-body force in a nonrelativistic system, probably motivated by the large two-nucleon scattering lengths in the $\mathrm{S}$ waves. He started with the Schrödinger equation with a short-range two-body potential and derived an effective three-body Schrödinger equation. He then noticed that there are an infinite number of three-body bound states with the ratios of binding energies of subsequent states being a universal constant. The result does not depend on the details of the shortrange two-body potential.

One may think that this phenomenon can be explained in the language of RG in an EFT. Such a formulation would make the universal feature of the phenomenon more transparent. Let us consider the EFT Lagrangian of a single nonrelativistic boson of mass $M$ :

$$
\begin{aligned}
\mathcal{L}= & \phi^{\dagger}\left(i \partial_{t}+\frac{\nabla^{2}}{2 M}\right) \phi-\frac{c_{0}}{4}\left(\phi^{\dagger}\right)^{2} \phi^{2} \\
& +\frac{c_{2}}{4}\left[\left(\phi^{2}\right)^{\dagger}\left(\phi \overleftrightarrow{\nabla}^{2} \phi\right)+\text { h.c. }\right] \\
& +\frac{b_{2}}{2}\left[\left(\phi^{2}\right)^{\dagger} \phi\left(i \partial_{t}+\frac{\nabla^{2}}{2 M}\right) \phi+\text { h.c. }\right]+\cdots \\
& -\frac{d_{0}}{36}\left(\phi^{\dagger}\right)^{3} \phi^{3}+\cdots,
\end{aligned}
$$

where $\overleftrightarrow{\nabla}^{2}=\overleftarrow{\nabla}^{2}-2 \overleftarrow{\nabla} \cdot \vec{\nabla}+\vec{\nabla}^{2}$ and the ellipses stand for the terms with more derivatives. Throughout this paper, we concentrate on the $\mathrm{S}$ waves, so that the operators contributing to higher partial waves are not included. We do not consider the operators which act on more than three-body states, e.g., $\left(\phi^{\dagger}\right)^{4} \phi^{4},\left(\phi^{\dagger}\right)^{5} \phi^{5}$, etc., because we are interested in the three-body sector and they do not contribute to the sector. Note that we have included the so-called redundant operators such as the one on the third line in Eq. (2.1), which are necessary to renormalize the theory off shell [26].

In an important paper, Bedaque, Hammer, and van Kolck 5] use another version of EFT with the so-called dimeron field, $D$. In the leading order the Lagrangian is 
given by

$$
\begin{aligned}
\mathcal{L}_{D}= & \phi^{\dagger}\left(i \partial_{t}+\frac{\nabla^{2}}{2 M}\right) \phi+g_{0} D^{\dagger} D \\
& +g_{1}\left[D^{\dagger} \phi^{2}+\left(\phi^{2}\right)^{\dagger} D\right]+g_{2} D^{\dagger} D \phi^{\dagger} \phi+\cdots
\end{aligned}
$$

They find the limit cycle behavior for $g_{2}$ from the scale invariant property of the three-body amplitude in the limit of an infinite scattering length,

$$
\begin{aligned}
H(\Lambda) & \equiv \frac{\Lambda^{2} g_{2}(\Lambda)}{4 M g_{1}^{2}(\Lambda)} \\
& =-\frac{\sin \left[s_{0} \ln \left(\Lambda / \Lambda_{*}\right)-\arctan \left(s_{0}^{-1}\right)\right]}{\sin \left[s_{0} \ln \left(\Lambda / \Lambda_{*}\right)+\arctan \left(s_{0}^{-1}\right)\right]}
\end{aligned}
$$

where $s_{0}$ is called the Efimov parameter, $s_{0}=$ $1.00624 \cdots, \Lambda$ is the floating cutoff, and $\Lambda_{*}$ is a constant.

Note, however, that although they find the intimate connection between the Efimov effect (as a consequence of discrete scale invariance) and the RG limit cycle behavior, they obtain the (floating) cutoff dependence of the coupling constant not from the RGEs. Note also that their analytic results are given only for the case of an infinite scattering length, and the case with a finite scattering length is considered only numerically.

More recently a field-theoretical derivation of the limit cycle behavior directly from the RGEs is given by Moroz, Floerchinger, Schmidt, and Wetterich [11]. They also use the dimeron field. Their formulation admits an arbitrary momentum dependence of the coefficient functions. In the leading-order approximation (the point-like approximation), the RGEs are solved analytically, but in the higher orders they are solved only numerically.

Even though the use of dimeron is useful, it is just an option. The RGEs and the limit cycle behavior should be obtained even if the dimeron field is not introduced. Such a demonstration would provide a better insight into the use of dimeron field.

\section{THE ONE-LOOP PROPERTY PUZZLE}

In this sections, we explain the one-loop puzzle and its solution. For simplicity, we consider a nonrelativistic bosonic system described by the Lagrangian (2.1). The dimeron field is not introduced.

\section{A. One-loop property of nonperturbative RGEs}

It is well known that nonperturbative RGEs have a kind of "one-loop" property. The Wegner-Houghton equation [19] for the Wilson action $S_{\text {eff }}$, for example,

$$
\begin{aligned}
\partial_{t} S_{\mathrm{eff}}= & \frac{1}{2 d t} \int_{p}^{\prime}\left\{\ln \left(\frac{\delta^{2} S_{\mathrm{eff}}}{\delta \phi_{p} \delta \phi_{-p}}\right)\right. \\
& \left.-\frac{\delta S_{\mathrm{eff}}}{\delta \phi_{p}}\left(\frac{\delta^{2} S_{\mathrm{eff}}}{\delta \phi_{p} \delta \phi_{-p}}\right)^{-1} \frac{\delta S_{\mathrm{eff}}}{\delta \phi_{-p}}\right\} \\
& +(\text { canonical scaling terms })
\end{aligned}
$$

has the "one-loop" term (the first line), together with the "dumbbell" term (the second line), where the integration with prime means the integration over shell mode, 1 $d t<p<1$. In the functional flow equation [21, 22],

$$
\partial_{t} \Gamma_{k}=\frac{1}{2} \operatorname{Tr}\left[\partial_{t} R_{k}\left(\Gamma_{k}^{(2)}[\phi]+R_{k}\right)^{-1}\right],
$$

where $\Gamma_{k}[\phi]$ is an averaged action with the momentumshell parameter $k$, on which the regularization function $R_{k}$ depends, $\Gamma_{k}^{(2)}$ is the second derivative of $\Gamma_{k}$ with respect to $\phi$, and $t \equiv \ln \left(k / \Lambda_{0}\right)$, the whole contributions come from the "one-loop" diagrams. In both cases, the "one-loop" diagrams are composed of the full propagators, so that the actual structure is much more complicated than it appears.

In nonrelativistic systems, because of the absence of antiparticles, the "one-loop" diagrams are really oneloop, i.e., the propagators are bare ones. There are no tadpole-type diagrams, in which an internal line starts and ends at the same vertex.

In the two-body sector, there is only one type of oneloop diagrams, with various kinds of vertices. It gives rise to the RGEs of the couplings of two-body contact interactions. Note that, since the two-body amplitude is given as the sum of bubble chains, the cutoff independence of the two-body amplitude also leads to the same RGEs.

For the theory under consideration, if the one-loop property persists in the three-body sector, there appear to be only three types of one-loop diagrams that contribute to the running of three-body coupling constants. See Fig. 1] It is clear that the RGE for $d_{0}$ would not exhibit periodic behavior. To be concrete, the RGE for $d_{0}$ would be

$$
\frac{d v}{d t}=-4 v+\alpha x^{3}-\beta v x
$$

where $x$ and $v$ are dimensionless coupling constants defined by

$$
x=\frac{M \Lambda}{4 \pi^{2}} c_{0}, \quad v=\frac{M \Lambda^{4}}{6\left(2 \pi^{2}\right)^{2}} d_{0},
$$

and $t=\ln \left(\Lambda_{0} / \Lambda\right)$ with $\Lambda$ being the floating cutoff, $\alpha$ and $\beta$ are positive dimensionless constants. In the limit of infinite scattering length, $x \rightarrow-1$, the RGE (3.3) can be easily solved but the periodic behavior cannot be obtained. Note that the inclusion of arbitrary momentum 

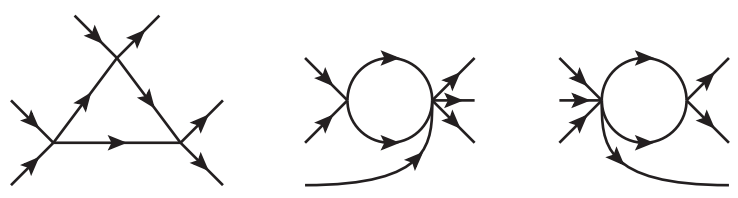

FIG. 1: Three types of time-ordered one-loop diagrams which are apparently the only diagrams contributing to the running of three-body coupling constants.

and energy dependent terms should not alter the conclusion given above because the Efimov effect, as a universality of long-distance physics, should be incorporated in the lowest orders in the derivative expansions of the effective field theory.

It seems that multi-loop diagrams must contribute to the RGE for $d_{0}$. But how?

\section{B. Normal-ordering}

It is important to note that, in nonrelativistic systems, the interaction operators are all (implicitly) assumed to be normal-ordered. Under this assumption, we can consider the sectors labeled by the particle number separately, and the $n$-body operators, such as $\left(\phi^{\dagger}\right)^{n} \phi^{n}$ do not affect the $m$-body sectors with $m<n$. This is a very favorable feature we would like to keep to simplify the calculations.

In order to make the operator structure explicit, let us consider an operator-formalism implementation of the Wilsonian RG transformation, where the field operator is Fourier transformed with the magnitude of momentum $p$ is bounded by the floating cutoff $(0 \leq p \leq \Lambda)$. The RG transformation amounts to the contractions of only the shell-modes $(\Lambda-\delta \Lambda<p \leq \Lambda)$ of the logarithm of the Dyson operator

$$
S_{\Lambda-\delta \Lambda} \equiv-i \ln \left[\left\langle T \exp \left[i S_{\Lambda}\right]\right\rangle_{\text {shell-mode contractions }}\right] .
$$

The field renormalization rescaling is unnecessary in our nonrelativistic theory. Diagrammatically it is represented as a sum of one-loop diagrams with the loop momenta being in the shell-mode and with the operators with lower-momentum modes being attached to the external lines.

The crucial point is that the counterterms generated by RG transformations are in general not normal-ordered. In order to obtain the correct RGEs for the coupling constants for the normal-ordered operators, one needs to rewrite the counterterms in the normal-ordered form. This rewriting of an $n$-body couterterm leads to normalordered $m$-body operators with $m \leq n$.

Let us explain what is going on by examples. The first example is the diagram shown in Fig. 2. With the loop momentum being in the shell $\Lambda-\delta \Lambda<p \leq \Lambda$, it generates an effective local interaction which can be canceled by 3-body counterterms that are all normal-ordered. Note

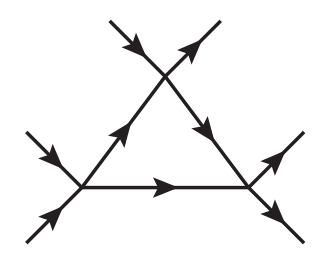

FIG. 2: A time-ordered diagram which contributes to threebody local counterterms when the loop momentum is set in the shell. The resulting counterterms are already in the normal-ordered form.

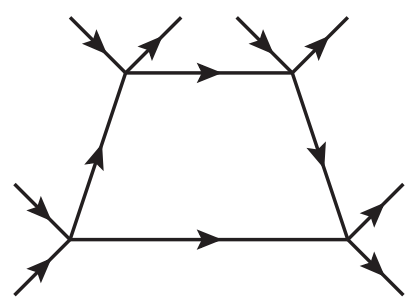

FIG. 3: A time-ordered diagram which contributes to fourbody local counterterms when the loop momentum is set in the shell. The resulting counterterms are not normalordered. The rewriting them in the normal-ordered form generates normal-ordered three-body operators as well as normalordered four-body operators.

that the diagram is a time-ordered one. It is impossible to contract any external outgoing and incoming lines without going backward in time. If we assign an external outgoing line and an incoming line with the creation and annihilation operators, $a^{\dagger}$ and $a$, respectively, this diagram has the $\left(a^{\dagger}\right)^{2}\left(a^{\dagger} a\right) a^{2}$ structure, which is already in the normal-ordered form. On the other hand, in the second example shown in Fig. 3 with the loop momentum being in the shell, the diagram generates the contributions that are not normal-ordered. In this case, it is possible to contract the external lines without going backward in time. In other words, the diagram has the $\left(a^{\dagger}\right)^{2}\left(a^{\dagger} a\right)\left(a^{\dagger} a\right) a^{2}$ structure which can be rewritten as a sum of $\left(a^{\dagger}\right)^{4} a^{4}$ and $\left(a^{\dagger}\right)^{3} a^{3}$. The latter contributes to the three-body sector, though naively the diagram appears to affect only the four-body sector. Note also that a contraction of the lines amounts to an additional loop, thus the latter contribution actually comes from the two-loop diagram.

When the diagram with the additional loop is considered, we should treat it in a Galilean invariant way. We need to impose the cutoff on the relative momentum of the two lines to maintain Galilean invariance. In this way, we realize that the "total momentum" of the loop must be the shell mode, while cutoff is imposed on the relative momentum, as shown in Fig. 4.

We go on to the third example in Fig. 5. The diagram has six incoming and six outgoing external lines. Naively it only produces effective six-body operators. However, since it is not normal-ordered, rewriting it in the normal- 


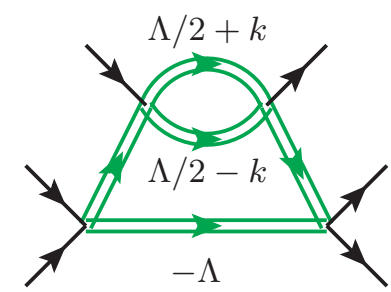

FIG. 4: The diagram obtained from Fig. 3 by normalordering. The external energies and momenta are set to zero for simplicity. The momenta are assigned so that the Galilean invariance is maintained. The double lines indicate that the propagators have momenta of order of the cutoff $\Lambda$. Note that all the momenta of the internal lines are related to the shell-mode momentum.

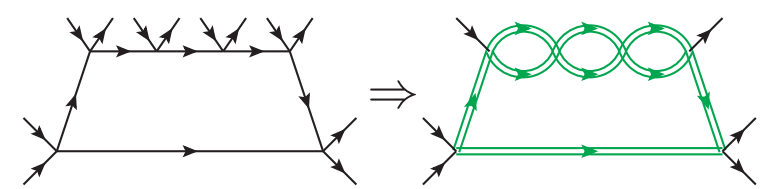

FIG. 5: A time-ordered diagram which contributes to sixbody local counterterms when the loop momentum is set in the shell. The resulting counterterms are not normal-ordered. The rewriting them in the normal-ordered form generates normal-ordered three-body operators, as well as operators which act only on other sectors. All the momenta of the lines of the additional loops are related to the shell mode.

ordered form is necessary to obtain the correct running of the coupling constants for the normal-ordered operators. There are several ways of contracting the lines. In particular, it gives rise to three-body operators, which actually come from four-loop diagrams because the contractions amount to loops. In a similar way, we can go on to one-loop diagrams with an arbitrary number of external lines. Again, Galilean invariance forces a particular momentum assignment so that the momenta of the lines of the additional loops are related to the shell mode.

There is another kind of diagrams generated by normal-ordering, in which the contractions give rise to internal lines which get across the shell-mode loop. See Fig. [6] for examples. Such diagrams do not contribute the RGEs. In the usual relativistic field theory, such diagrams can be obtained after constructing the effective local vertex by connecting the legs. That is one of the reasons why the RGEs have the "one-loop" property mentioned above. In the nonrelativistic field theory, because there is no antiparticle, tadpole-type diagrams cannot occur. We can just disregard these contractions. The crucial difference between the diagrams considered in the previous paragraphs and the ones with lines across the shell-mode loop is that in the former the additional lines are forced to related to the shell mode, while in the latter they are not.

The analysis given above provides the solution of the
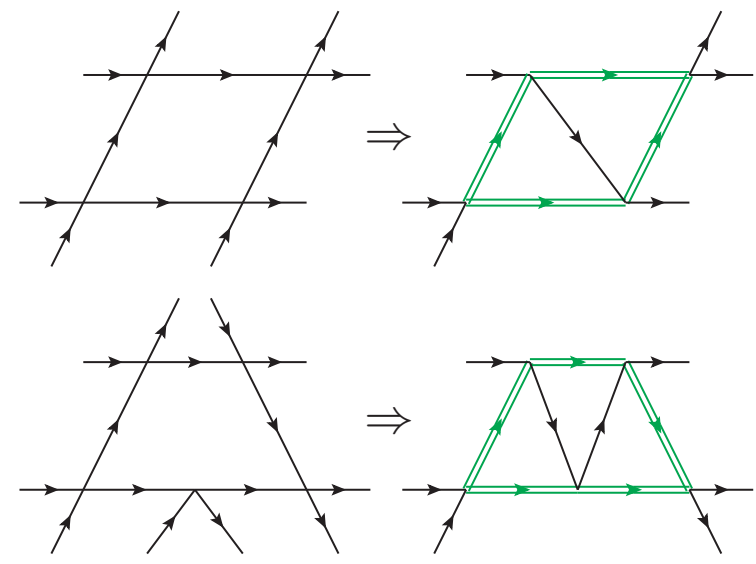

FIG. 6: Examples of the diagrams in which the additional lines get across the shell-mode loop. The momentum associated with these lines are not related to the shell mode.

puzzle. The one-loop property persists even in the threebody sector, but the generated diagrams are not in general in the normal-ordered form. In order to obtain the running of the coupling constant for the operators in the Lagrangian of a nonrelativistic theory, one needs to rewrite the contributions in the normal-ordered form because the operators in the Lagrangian are usually assumed to be normal-ordered. In this rewriting, some contractions may be needed. A contraction amounts to an additional loop. The diagrams obtained by normalordering in which all the lines of the additional loop are forced to be related to the shell mode, with an arbitrary number of loops, may contribute to the running of threebody operators.

Note that no multi-loop contribution can occur in the two-body sector. This is a special feature of the two-body sector. On the other hand, it is much more involved in many-body sectors. In the three-body sector, as we will see, even though infinitely many diagrams contribute to the running of the three-body coupling $d_{0}$, they can be resummed.

\section{Breakdown of the naive one-loop property in nonrelativistic theory}

In this section, we consider the results obtained in the previous section from a different side. Let us start with the reason why the one-loop property arises in the usual formulation of nonperturbative RGEs.

Consider the cutoff dependence of a diagram with an arbitrary number of loops, whose momenta are cutoff at $\Lambda$. The differentiation with respect to the cutoff $\Lambda$ picks up the shell mode of each of the loops, and the result is a sum of the diagrams, in each of which only one of the loops has the shell-mode momentum.

Thus, if we have a counterterm which has the same cutoff dependence with opposite sign as that of the loop 


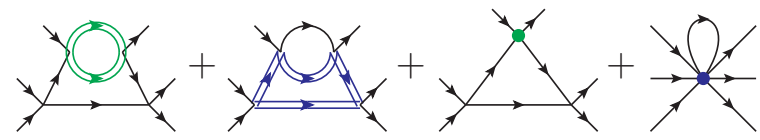

FIG. 7: The shell-mode diagrams of a two-loop example and the corresponding diagrams with the shell-mode loop is replaced with the counterterm in the relativistic theory.

in the shell mode, the addition of the diagram in which each loop of the original diagram is replaced with the corresponding counterterm cancels the cutoff dependence of the loop. Note that the diagram with the counterterm has a reduced number of loops. One can proceed iteratively and determine all the counterterms which are necessary to make the original diagram finite. This is a physical picture of how the one-loop property arises.

Let us consider a simple two-loop example shown in Fig. 7 to illustrate how the above procedure goes in a relativistic theory. The loop momenta are cutoff at $\Lambda$. (We have to work in Euclidean space and impose the condition on the magnitude of the four-momentum of each propagator in order to maintain Lorentz (rotational) invariance.) The differentiation of the amplitude with respect to $\Lambda$ is given by two terms: each has a single loop whose momentum is in the shell. The point is that we can consider each loop and the corresponding counterterm individually. The cutoff dependence of each loop can be compensated by adding the diagram with the corresponding counterterm. Note that the fourth diagram in Fig. 7 contains the tadpole loop. As we explained in the previous paragraph, this tadpole contribution is required to make the amplitude cutoff independent.

Let us now suppose the same diagram arises in a nonrelativistic theory. Apart from the fact that there is no way to maintain Galilean invariance by imposing a cutoff on the magnitude of the three-momentum of each propagator, we have a trouble with the tadpole: because of the absence of anti-particle, there are no tadpole contributions so that we cannot make the amplitude cutoff independent. This is the reason why the naive oneloop property must be broken in nonrelativistic theory: the counterterms obtained by one-loop diagrams do not renormalize the theory.

A careful examination of the cutoff dependence of multi-loop diagrams in nonrelativistic theory shows that the loops cannot always be treated individually because of Galilean invariance. There are contributions in which more than one loops should be simultaneously in the shell mode. They compensate the lack of the tadpole contributions absent in the nonrelativistic theory. Such contributions are obtained by the procedure described in the previous section.

Note also that the cutoff dependence of the multi-loop diagrams in Fig 6 can be compensated by the counterterms of the individual loops, because the internal lines are not related by the symmetry so that the loop momenta do not need to be the shell mode simultaneously.

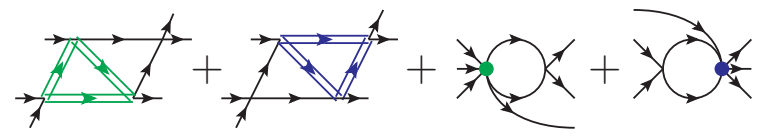

FIG. 8: The cutoff dependence of the two-loop diagram considered in Fig. 6 can be compensated by the counterterms of the individual loops.

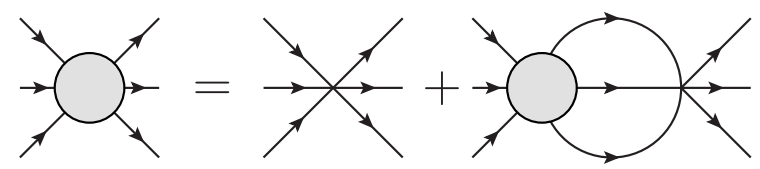

FIG. 9: The Lippmann-Schwinger equation for the three-body amplitude in the case where only the three-body interaction is present.

\section{See Fig. 8}

There is another simple way to see that the naive oneloop property of RGEs should break down in nonrelativistic theory. Consider the case where only the $\left(\phi^{\dagger}\right)^{3} \phi^{3}$ interaction is present. In this case, two-body interactions are not generated (because of the absence of the tadpole contributions) and the three-body amplitude is given by a sum of the chains of the two-loop diagrams, depicted in Fig. 9. In order to renormalize the amplitude, one needs the counterterm for the two-loop diagram. The one-loop diagram does not give rise to such a counterterm because the tadpole cannot contribute in the nonrelativistic theory. Note that the momenta of the two loops must be in the shell simultaneously because of the symmetry among the three propagators and cannot be treated individually.

\section{DERIVATION OF RGES}

As we explained in the previous section, there are multi-loop contributions to three-body operators obtained by normal-ordering rewriting of the one-loop diagrams that are not in the normal-ordered form. It turns out that there are five types of such "normal-ordered oneloop" (NOOL) diagrams, together with two genuine (i.e., already normal-ordered) one-loop ones. They are given in Fig.10 Note that the multi-loop contributions are neatly written in terms of the two-body scattering amplitude (a blob in Fig. 10) which satisfies the Lippmann-Schwinger equation depicted in Fig. 11. Note also that the diagram in Fig. 2 appears as a part of the first diagram in Fig. 10.

The RGEs can be obtained by setting the loop momenta of the seven diagrams in the shell and canceling their cutoff dependence by adding suitable three-body counterterms, as we depicted in Fig. 12 ,

It is important to notice that a sum of additional loops,

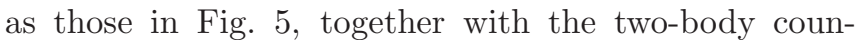
terterms, forms the cutoff-independent two-body amplitude so that it does not contribute to the running of the coupling constants of the three-body operators. Simi- 

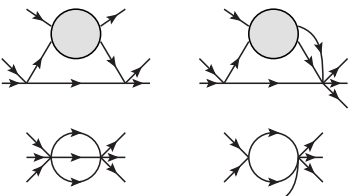
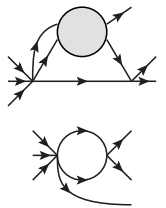
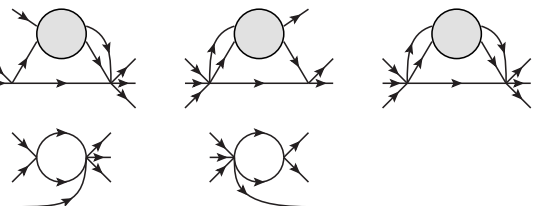

FIG. 10: The NOOL diagrams together with the two genuine one-loop diagrams. The shaded blob stands for the two-body amplitude defined in Fig. 11

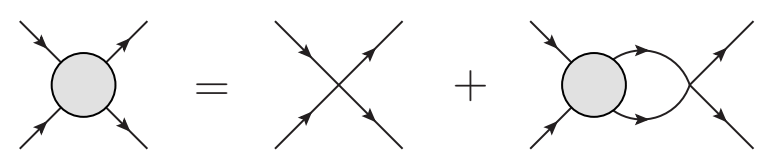

FIG. 11: The Lippmann-Schwinger equation for the two-body scattering amplitude, represented as a shaded blob. The vertex actually stands for a collection of vertices, $c_{0}, c_{2}$, and $b_{2}$ in the present approximation.

larly, the cutoff dependence of the additional loops on the oblique sides of the trapezoids in the second, third, and the fourth diagrams in Fig. 12 is already taken into account by the sixth and the seventh diagrams in Fig. 12,

In this section, we consider only the terms that are explicitly shown in Eq. (2.1), that is, two-body operators up to including $\mathcal{O}\left(p^{2}\right)$ and a three-body operator without derivatives. This truncation makes our RGEs approximate. Higher order corrections are discussed in Sec. VI.

The two-body amplitude can be treated separately. We assume that the cutoff $\Lambda$ is small enough so that the twobody amplitude is given in the effective range expansion form. The renormalized off-shell amplitude may be written as

$\mathcal{A}\left(\mu, \boldsymbol{k}_{1}^{2}, \boldsymbol{k}_{2}^{2}\right)=\frac{8 \pi}{M}\left[\frac{1}{a_{2}}-\mu+\frac{1}{2} r_{e} \mu^{2}-\frac{3 h}{a_{2}}\left(2 \mu^{2}+\boldsymbol{k}_{1}^{2}+\boldsymbol{k}_{2}^{2}\right)\right]^{-1}$,

where $a_{2}$ and $r_{e}$ are the scattering length and the effective range respectively, $\boldsymbol{k}_{1}$ and $\boldsymbol{k}_{2}$ are incoming and outgoing relative momenta,

$$
\mu=\sqrt{-M P^{0}+\boldsymbol{P}^{2} / 4-i \epsilon},
$$

with $P^{\mu}=\left(P^{0}, \boldsymbol{P}\right)$ being the total momentum. (Note that Eq. (4.1) is different by a symmetric factor $1 / 2$

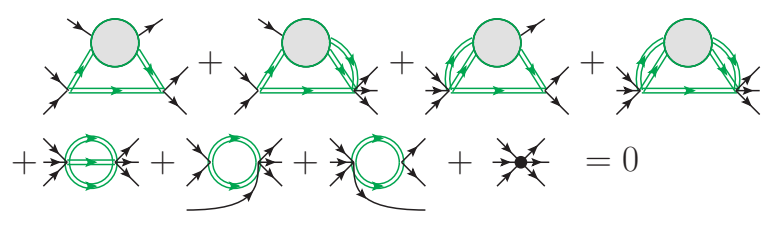

FIG. 12: A diagrammatic representation for the RGEs of the three-body operators. Propagators with the momentum being in the shell are denoted by double lines. from that for the "spinless nucleon" given in Ref. 26] for which, to mimic the nucleon case, we intentionally ignore the factor.) We have an off-shell parameter $h$ coming from the redundant operator. Note that in the on-shell amplitude, the third term vanishes. The parameters $a_{2}$, $r_{e}$, and $h$ are cutoff independent.

The RGEs for the coupling constants for the two-body operators can be obtained in Ref. [26]:

$$
\begin{aligned}
\frac{d X}{d t} & =(1-X)\left(Y+3 X^{2}\right) / X \\
\frac{d Y}{d t} & =-Y\left(6 X^{3}-5 X^{2}+2 X Y-Y\right) / X^{2} \\
\frac{d Z}{d t} & =-\left(6 X^{3} Z-3 X^{2} Z+2 X Y Z+Y^{2}\right) / X^{2}
\end{aligned}
$$

where $X, Y$, and $Z$ are combinations,

$$
\begin{aligned}
& X=1+\frac{1}{3}(y+z), \\
& Y=x-\frac{1}{5}(y+z)^{2}, \\
& Z=2 y+\frac{1}{3}(y+z)^{2},
\end{aligned}
$$

of the dimensionless coupling constants,

$$
x=\frac{M \Lambda}{4 \pi^{2}} c_{0}, \quad y=\frac{M \Lambda^{3}}{4 \pi^{2}} 4 c_{2}, \quad z=\frac{\Lambda^{3}}{4 \pi^{2}} b_{2} .
$$

The parameter $t$ is defined as $t=\ln \left(\Lambda_{0} / \Lambda\right)$, where $\Lambda_{0}$ is the physical cutoff which is the limit of the applicability of the EFT, and $\Lambda$ is the floating cutoff. There is a nontrivial fixed point, $\left(X^{*}, Y^{*}, Z^{*}\right)=(1,-1,-1)$, besides the trivial one, $(1,0,0)$.

These RGEs have an analytic solution:

$$
\begin{aligned}
& X=\frac{(C \Lambda)^{-1}-1}{(C \Lambda)^{-1}-1-C^{\prime} \Lambda^{2}}, \\
& Y=\frac{(C \Lambda)^{-1}-1}{\left[(C \Lambda)^{-1}-1-C^{\prime} \Lambda^{2}\right]^{2}}, \\
& Z=\frac{C^{\prime \prime} \Lambda-1}{\left[(C \Lambda)^{-1}-1-C^{\prime} \Lambda^{2}\right]^{2}},
\end{aligned}
$$

where $C, C^{\prime}$, and $C^{\prime \prime}$ are the integration constants. They are related to the effective range expansion parameters, $a_{2}, r_{e}$, and $h$, by

$$
C=\frac{2 a_{2}}{\pi}, \quad C^{\prime}=h, \quad C^{\prime \prime}=\frac{\pi}{4} r_{e} .
$$

In terms of them, the three-body RGEs are written as follows:

$$
\begin{aligned}
\frac{d v}{d t}= & {\left[3 b^{2} \frac{1}{S^{2}}\left(T-\frac{2}{b} U\right)-c\right] v^{2} } \\
& +\left[12 b \frac{V}{S^{2}}\left(T-\left(3+\frac{1}{b}\right) U\right)-4-6 V\right] v \\
& +12 \frac{V^{2}}{S^{2}}(T-6 U)
\end{aligned}
$$


where we have introduced several combinations,

$$
\begin{aligned}
S & =X^{2}-a Y, \\
T & =Y S+\frac{3}{4}\left(X^{2} Z+Y^{2}\right), \\
U & =X(X-1)\left(X^{2}+Y\right), \\
V & =Y+3(X-1)+\frac{9}{5}(X-1)^{2},
\end{aligned}
$$

together with numerical constants,

$$
\begin{aligned}
a & =\frac{\sqrt{3}}{4} \pi-1, \\
b & =1-\frac{\sqrt{3}}{4} \pi+\frac{\sqrt{3}}{2} \arctan \frac{\sqrt{3}}{2}, \\
c & =\frac{7}{3}-\frac{\sqrt{3}}{4} \pi-\frac{7 \sqrt{3}}{18} \arctan \frac{\sqrt{3}}{2} .
\end{aligned}
$$

Note that in these equations, $X, Y$, and $Z$ are the solution, Eq. (4.10), with the integration constants, Eq. 4.11.

In Appendix B a sample calculation of a contribution to the RGE is given for the purpose of illustration. It is a bit difficult to find out the shell mode contribution of the two-loop diagram shown in the fifth diagram in Fig. 12. In Appendix C. we explain how to identify the shell mode contribution.

It is important to note that the RGE now has terms proportional to $v^{2}$. The appearance of these terms allow the periodic behavior of $v$ in the RG evolution, as we will see in the next section.

It is interesting to note that these seven diagrams in Fig. 10 are the irreducible building blocks of which the three-body amplitude is composed, just as simple twopoint bubbles are the building blocks of the two-body amplitude.

The amplitude composed of these blocks can be compared with that obtained in the formulation with the dimeron field. The two-body amplitude is equal to the (dressed) dimeron propagator multiplied by the factor $g_{1}^{2}$ coming from the two-particle-dimeron vertices at the ends. See Fig. 13. With this identification, one easily sees the correspondence between the three-body amplitude in our formulation and that in the formulation with dimeron.

There is however a difference: in our formulation, a one-loop diagram with a two-body vertex and a threebody vertex (which we call a "two-three loop") contributes, while in the existing calculations with dimeron these contributions are not included.

Note that the cutoff dependence of the two-three loop cannot be renormalized as a part of two-body amplitude because the two-body amplitude is composed solely of two-body vertices, but is canceled by three-body counterterms, as we explained earlier. See the sixth and the seventh diagrams in Fig. 12, The momentum dependence becomes important when the two-three loop is embedded in the three-body amplitude. When the shell-mode momentum is considered as in the second, third, and the

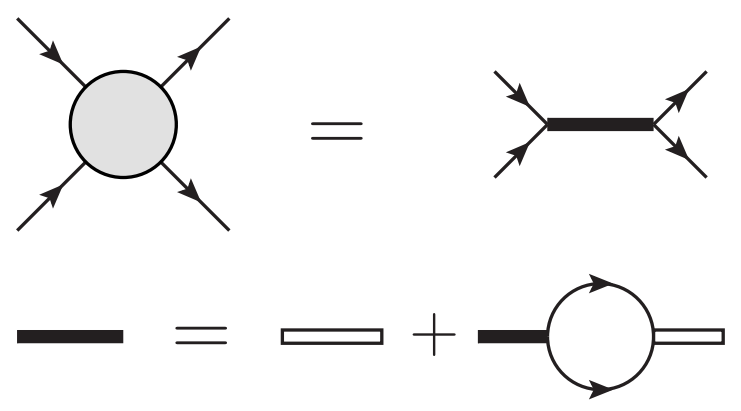

FIG. 13: The dimeron representation of the two-body amplitude, composed of the dressed dimeron propagator and the two-particle-dimeron vertices at the ends.

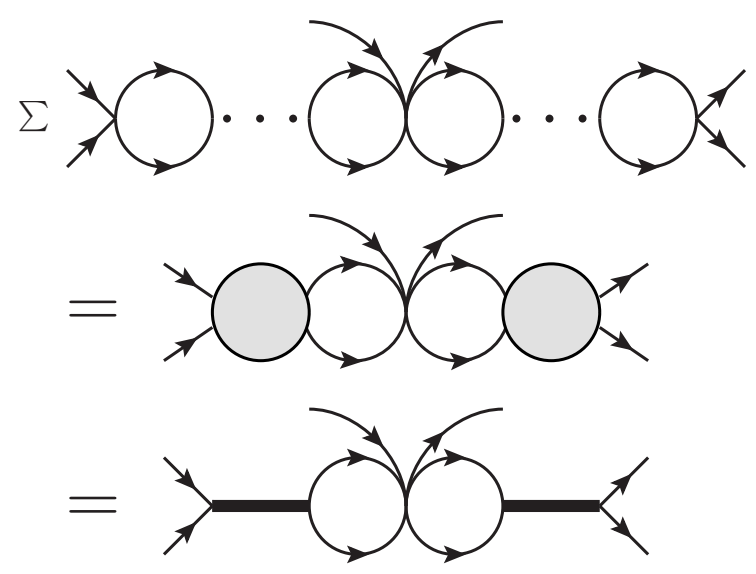

FIG. 14: The insertion of the three-body vertex in the twobody amplitude. In the third line, the two-body amplitudes are replaced by the full dimeron propagator. Because of the two-three loops, it is not equal to the (momentumindependent) $D \phi-D \phi$ vertex.

fourth diagrams in Fig. 12 the momentum dependence of the two-three loops becomes an additional source of the cutoff dependence.

In the existing calculations with dimeron, the $D \phi$ $D \phi$ vertex is momentum independent even for the full dimeron lines. On the other hand, as depicted in Fig. [14, a single insertion of the three-body vertex in the twobody amplitude cannot be represented by a momentumindependent $D \phi-D \phi$ vertex, but has momentum dependence due to the two-three loops.

\section{RG FLOWS AND THE EFIMOV PARAMETER}

A. Flows in the $r_{e}=h=0$ subspace

Let us begin, for simplicity, with the RG flows on which the effective range, $r_{e}$, and the off-shell parameter, $h$, are zero. In this case, the RGEs are drastically simplified 


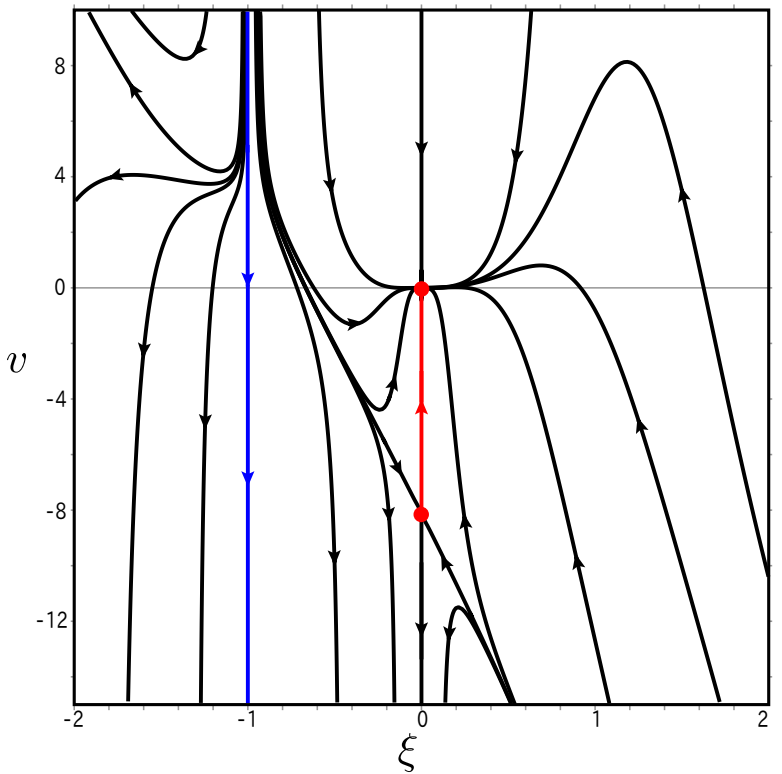

FIG. 15: The RG flow in the $\xi-v$ plane. The arrows indicate the directions of the flow to the infrared (larger $t$ ).

and are written in terms of $\xi \equiv Y / X^{2}$, as

$$
\begin{aligned}
\frac{d}{d t} \xi= & -\xi(1+\xi) \\
\frac{d}{d t} v= & \frac{12 \xi^{3}}{1-a \xi}+\left(\frac{12 b \xi^{2}}{1-a \xi}-4-6 \xi\right) v \\
& +\left(\frac{3 b^{2} \xi}{1-a \xi}-c\right) v^{2}
\end{aligned}
$$

Note that the RGE for $\xi$ is the same as that for $x$ in the leading order approximation. The RG flow in the $\xi-v$ plane are shown in Fig. 15. The line $\xi=-1$ corresponds to the nontrivial fixed point of the two-body RGEs. The flow there goes down periodically, exhibiting the limit cycle behavior. In addition to the trivial fixed point, we find a nontrivial fixed point $\left(\xi_{\star}, v_{\star}\right)=(0,-4 / c)=$ $(0,-8.126 \cdots)$, which we call the Borromean fixed point, though it apparently has nothing to do with the known Borromean systems. In the full set of RGEs, the Borromean fixed point is at $\left(x_{\star}, y_{\star}, z_{\star}, v_{\star}\right)=(0,0,0,-4 / c)$, so that there are no two-body interactions but only the three-body interaction.

One may notice that there is a singularity in Eq. (5.2) at $\xi=1 / a$. This is due to the pole of the two-body amplitude which corresponds to the bound state. See Appendix D for more details.

\section{B. Limit cycle}

The limit cycle behavior is due to the $v^{2}$ term in the RGE for $v$. With $(x, y, z)=(-1,-1 / 2,1 / 2)((X, Y, Z)=$ $(1,-1,-1))$, where, $a_{2}=\infty$ and $r_{e}=h=0$, the RGE for $v$ becomes

$$
\frac{d v}{d t}=A+B v+C v^{2}
$$

with

$$
A=-\frac{48}{\sqrt{3} \pi}, B=2+\frac{48 b}{\sqrt{3} \pi}, C=-\frac{12 b^{2}}{\sqrt{3} \pi}-c,
$$

so that the discriminant is negative,

$$
D \equiv B^{2}-4 A C=-4.27374 \cdots<0 .
$$

Thus the solution is given by

$$
v=\frac{-B+\sqrt{-D} \tan \left(\frac{\sqrt{-D}}{2}\left(t+t_{0}\right)\right)}{2 A},
$$

where $t_{0}$ is a constant of integration. It shows the periodic behavior with the period

$$
T=\frac{2 \pi}{\sqrt{-D}} \simeq 3.03932 .
$$

In terms of the Efimov parameter, $s_{0}$, it is written as

$$
s_{0}=\frac{\pi}{T} \simeq 1.03365
$$

which is only $2.7 \%$ off the Efimov's value.

It is interesting to compare the value with that obtained in the "point-like" approximation in the dimeron formulation [11], $s_{0} \simeq 1.393$, which is about $40 \%$ off the Efimov's value. The difference is due to the contributions from the two-three loops, as we explained in the previous section.

\section{Off-critical cases}

We can investigate the off-critical, i.e., finite scattering length, behavior. Fig. 16 and Fig. 17 show the running of $v$ and $\xi$ as functions of $t$ starting with $\xi(t=0)+$ $1=-0.001$ and $\xi(t=0)+1=0.001$, respectively. If the flow is close enough to the critical line $(\xi=-1), v$ diverges (to negative infinity) a finite number of times before going to the trivial fixed point (for $\xi>-1$ ) or to the positive infinity (for $\xi<-1$ ). We suspect that the occurrence of divergences corresponds to the existence of three-body bound states, as for the critical case in which the flow diverges infinite times, corresponding to an infinite number of bound states. Thus, if the flow is close enough to the critical line, there are a finite number of three-body bound states.

The behavior in the two-body strong-coupling phase $(\xi<-1)$ is particularly interesting from the NEFT point of view. In the ${ }^{3} S_{1^{-}}{ }^{3} D_{1}$ channel, the two-nucleon system is in the strong-coupling phase and very close to the critical case. There is a shallow two-body bound state, deuteron. The binding energy of the triton $\left(E_{t}=\right.$ 


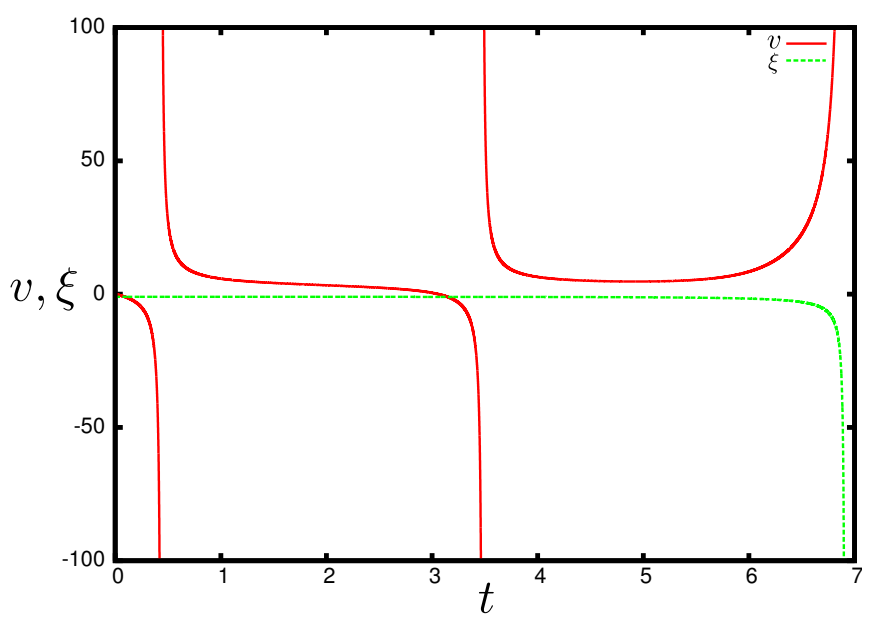

FIG. 16: The running of $v$ and $\xi$ as functions of $t$ in the twobody strong-coupling phase $(\xi<-1)$. The initial value is taken as $v(t=0)=0$ and $\xi(t=0)=-1.001$.

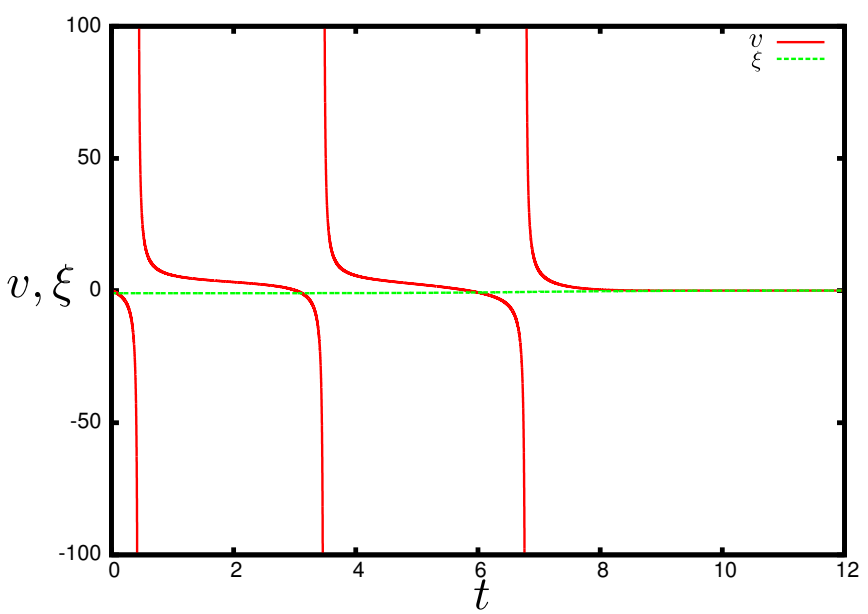

FIG. 17: The running of $v$ and $\xi$ as functions of $t$ in the twobody weak-coupling phase $(\xi>-1)$. The initial value is takes as $v(t=0)=0$ and $\xi(t=0)=-0.999$.

8.48 MeV) which is in the spin one-half channel in the neutron-deuteron system, is larger than that of deuteron $\left(E_{d}=2.2246 \mathrm{MeV}\right)$. Even though the present theory is bosonic, we expect that the same RG structure persists in the fermionic theory, and that the interplay between the three-body and the two-body bound states of the bosonic theory may explain that of the nucleon systems.

The coupling $\xi$ diverges to negative infinity at a finite value of $t\left(t_{2}\right)$ in the two-body strong-coupling phase. It implies the existence of the two-body bound state and the value $t_{2}$ would correspond to the binding energy of it. Once $\xi$ diverges to negative infinity, the flow appears from the positive infinity of $\xi$, decreasing to the trivial fixed point, $\xi=0$. (The continuity may be easily seen by introducing a change of variable from $\xi$ to $\theta \equiv \tan ^{-1} \xi$. The RGE (5.1) becomes $d \theta / d t=-\sin \theta(\cos \theta+\sin \theta)$. The transition from the negative infinity to the positive

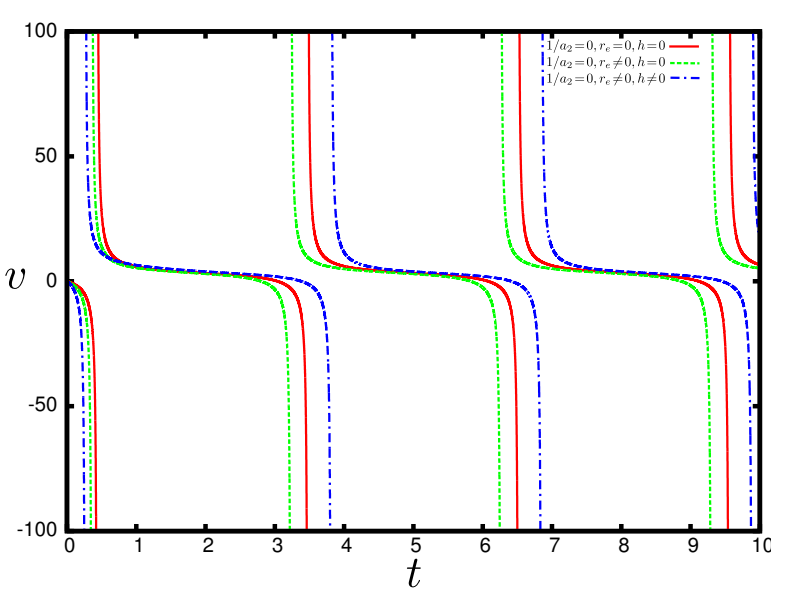

FIG. 18: The effects of nonzero values of $h$ and $r_{e}$. The red line is the solution Eq. (5.6), with $r_{e}=h=0$. The green line is the case with $r_{e} \Lambda_{0}=1$ and $h=0$, where $\Lambda_{0}$ is the physical cutoff. The blue line is the case with $r_{e} \Lambda_{0}=h \Lambda_{0}^{2}=1$. Three of them have asymptotically the same period.

infinity of $\xi$ corresponds to passing $-\pi / 2$ of $\theta$.)

When $v$ diverges to negative infinity in the two-body strong-coupling phase $(\xi<-1)$, it occurs before $\xi$ diverges. In the positive $\xi$ region, on the other hand, we numerically find, by examining the flow, that all the flows which come from the two-body strong-coupling phase never diverge to negative infinity. The above observation implies that, if both the two-body and the three-body bound states exist, the binding energy of the two-body bound state is always smaller than the three-body bound state.

\section{Borromean fixed point}

To our best knowledge, the existence of the Borromean fixed point has never been noticed in the literature. The existence of it and of the critical surface on which the Borromean fixed point resides implies a new phase where the two-body interactions do not support the two-body bound states, but the strong three-body interaction gives rise to the three-body bound states.

We linearize the RGE near the fixed point, $(\xi, v)=$ $\left(\xi_{\star}, v_{\star}\right)+(\delta \xi, \delta v)$,

$$
\frac{d}{d t}\left(\begin{array}{c}
\delta \xi \\
\delta v
\end{array}\right)=\left(\begin{array}{cc}
-1 & 0 \\
\frac{24}{c}\left(1+\frac{2 b^{2}}{c}\right) & 4
\end{array}\right)\left(\begin{array}{l}
\delta \xi \\
\delta v
\end{array}\right)
$$

By diagonalizing it, we see that the scaling dimension of the relevant coupling is +4 . In the next section, we investigate the Borromean fixed point once again with higher order corrections. 


\section{E. Effects of nonzero values of $r_{e}$ and $h$}

Going back to the full set of RGEs, Eq. 4.12 together with Eqs. (4.10), we can investigate the effects of nonzero values of $h$ and $r_{e}$. The results are shown in Fig. 18. It is interesting to note that they affect the flow of $d_{0}$, especially the "period," even though their effects damp as $t$ grows. Physically speaking, it implies that the ratios of the binding energies of the three-body bound states varies and asymptotically becomes the universal value $e^{-2 \pi / s_{0}}$ for the bound states accumulate toward the threshold. It is surprising to see that the parameter, $h$, corresponding to the redundant operator has such observable effects, even though it does not contribute to the two-body observables. Furthermore, it does not even appear in the off-shell amplitude, Eq. (4.1), in the critical case $\left(1 / a_{2}=0\right)$ !

\section{HIGHER ORDER CORRECTIONS}

In this section, we concentrate on the cases in which the two-body sector is on the nontrivial and the triv- ial fixed points, and consider the effects of higher order three-body operators. Note that all the irrelevant twobody operators do not contribute in these cases.

We include the following three-body operators,

$$
\begin{aligned}
\mathcal{L}_{\text {higher }}=\frac{d_{2}}{48} & {\left[\left(\phi^{3}\right)^{\dagger} \phi\left(\phi \overleftrightarrow{\nabla}{ }^{2} \phi\right)+\text { h.c. }\right] } \\
& +\frac{e_{2}}{24}\left[\left(\phi^{3}\right)^{\dagger} \phi\left\{\phi\left(i \partial_{t}+\frac{\nabla^{2}}{2 M}\right) \phi\right\}+\text { h.c. }\right],
\end{aligned}
$$

and obtain the RGEs for the dimensionless coupling constants, $v, s$, and $w$, the latter two of them are defined as

$$
u=\frac{M \Lambda^{6}}{\left(2 \pi^{2}\right)^{2}} d_{2}, \quad w=\frac{\Lambda^{6}}{\left(2 \pi^{2}\right)^{2}} e_{2}, \quad s=u+\frac{1}{3} w .
$$

\section{A. On the nontrivial fixed point}

On the nontrivial fixed point of the two-body sector, $(x, y, z)=(-1,-1 / 2,1 / 2)$, RGEs are given by

$$
\begin{aligned}
\frac{d v}{d t}= & -\frac{48}{\sqrt{3} \pi}+\left(2+\frac{48 b}{\sqrt{3} \pi}\right) v-\left(\frac{12 b^{2}}{\sqrt{3} \pi}+c\right) v^{2}+\left(\frac{3}{2}+\frac{4}{\sqrt{3} \pi}\right) s-\left(\frac{7}{120}+\frac{1}{12 \sqrt{3} \pi}\right) s^{2}-\left(\frac{2 b}{\sqrt{3} \pi}+\frac{1}{3}\right) v s, \quad(6.3 \\
\frac{d s}{d t}= & \frac{64}{3 \sqrt{3} \pi}-\left(2-\frac{24 b}{\sqrt{3} \pi}+\frac{8}{9 \sqrt{3} \pi}\right) s-\left(\frac{1}{6}+\frac{b}{\sqrt{3} \pi}\right) s^{2}-\left(2+\frac{32 b}{3 \sqrt{3} \pi}\right) v-\left(\frac{12 b^{2}}{\sqrt{3} \pi}+c\right) v s, \\
\frac{d w}{d t}= & \frac{576}{\sqrt{3} \pi}+\frac{48 b}{\sqrt{3} \pi} w-24\left[1+\frac{8}{\sqrt{3} \pi}\left(b+\frac{4}{7}\right)\right] v-\left[6+\frac{8}{\sqrt{3} \pi}(9 b+4)\right] s+6\left[e+\frac{8 b}{\sqrt{3} \pi}(b+3 d)\right] v^{2} \\
& +\left[\frac{1}{4}+\frac{4}{\sqrt{3} \pi}\left(\frac{3}{4} b+\frac{1}{12}\right)\right] s^{2}+\left[3 c+\frac{4}{\sqrt{3} \pi}\left(9 b^{2}+2 b+3 d\right)\right] v s-2\left(\frac{12 b^{2}}{\sqrt{3} \pi}+c\right) v w-\left(\frac{2 b}{\sqrt{3} \pi}+\frac{1}{3}\right) s w
\end{aligned}
$$

where the constants $b, c$ are given in Eqs. (4.17) and $d$ and $e$ are defined as

$$
\begin{aligned}
& d=-\frac{2}{7}+\frac{\sqrt{3}}{6} \pi-\frac{\sqrt{3}}{3} \arctan \frac{\sqrt{3}}{2}, \\
& e=-\frac{2}{3}+\frac{\sqrt{3}}{6} \pi+\frac{\sqrt{3}}{9} \arctan \frac{\sqrt{3}}{2} .
\end{aligned}
$$

Note that RGEs for $v$ and $s$ do not depend on $w$, so that they can be solved without solving the RGE for $w$. In the following we mainly consider the flows in the $v$-s plane.

These RGEs can be solved numerically. The $t$ dependence of the coupling constants, $v, s$, together with the leading order one for $v$, is shown in Fig. 19. Note that after an initial transient region, they exhibit periodic behavior. Note also that they do not diverge in the periodic region. The RG flow in the $v$-s plane is shown in Fig. 20.
We numerically read off the period of the limit cycle as 2.9810, which corresponds to the value of Efimov parameter $s \simeq 1.0539$. The value is about $4.73 \%$ off the Efimov's value. Compared with the leading order result, it deviates a bit more from the Efimov's value, though it is still very close. It might suggest that the convergence is not monotonous.

It is interesting to see that the limit cycle behavior emerges very differently from the leading-order one discussed in the previous section. In the leading order, since there is only one coupling, the periodicity is not possible unless it gets through the infinity. On the other hand, in the higher order, it is possible for the flows to have a periodicity while staying finite. It explains why the drastic change of the behavior of the RG flows occurs when the higher order contributions are taken into account. 


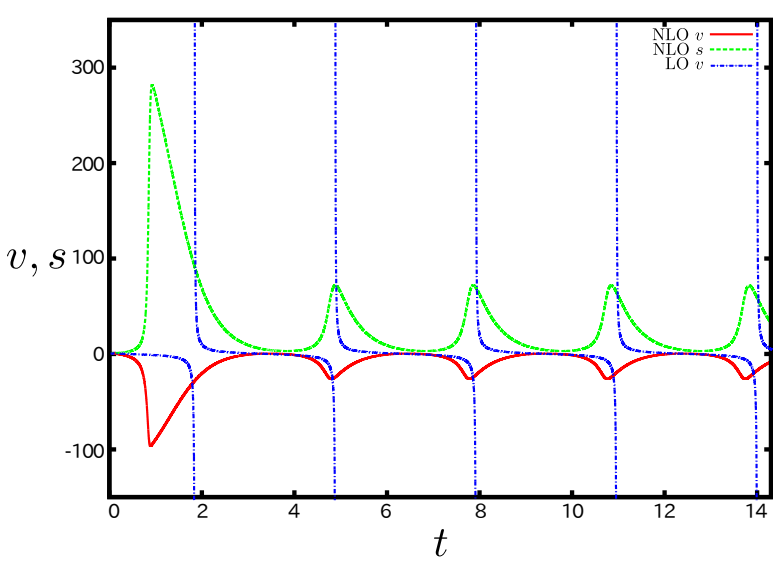

FIG. 19: The $t$ dependence of the three-body coupling constants, $v$ and $s$. The blue line stands for the leading order solution of $v$. The initial values are $\left(v_{0}, s_{0}\right)=(0.9,0.7)$.

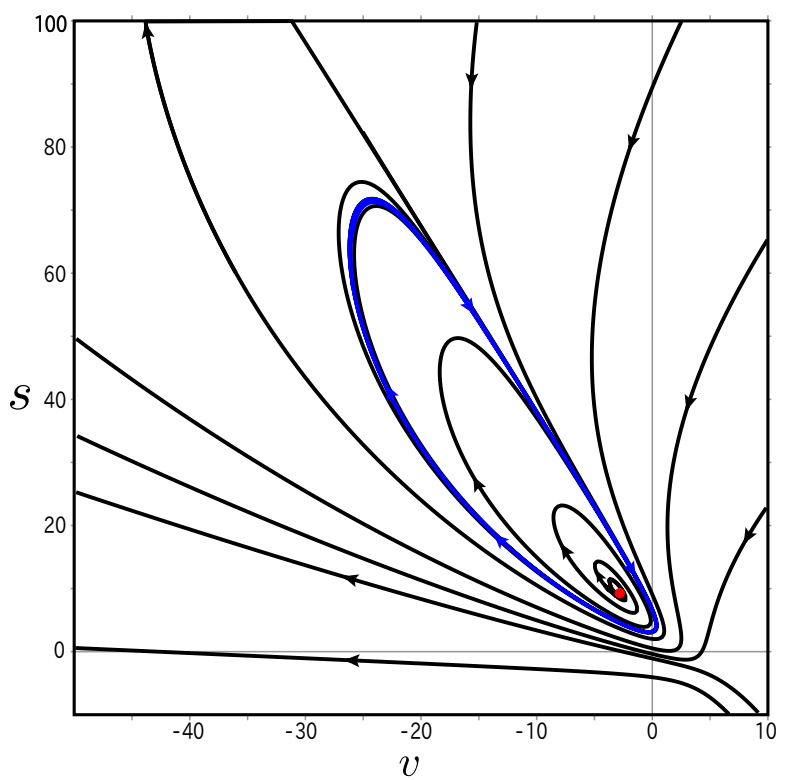

FIG. 20: The limit cycle behavior in the $v$-s plane. The blue loop is the limit cycle. Inside the loop there is a nontrivial fixed point (red bullet).

Another difference comes from the existence of the transient region. In the leading order, exact periodicity appears from the beginning. Actually the structure of the RG flow in the higher order is much richer. First of all, there is a nontrivial fixed point, numerically found to be at $\left(v_{\star}, s_{\star}, w_{\star}\right)=(-3.14,10.05,-60.73)$. As shown in Fig. 20, the flows from this nontrivial fixed point gradually approach to the limit cycle. On the other hand, the flows outside the limit cycle loop are grouped in the two categories: some flows are directly attracted by the limit cycle and gradually approach to it, and the others go to infinity (and come back from the opposite side) and then approach to the limit cycle. The existence of these two categories is related to another nontrivial fixed point at

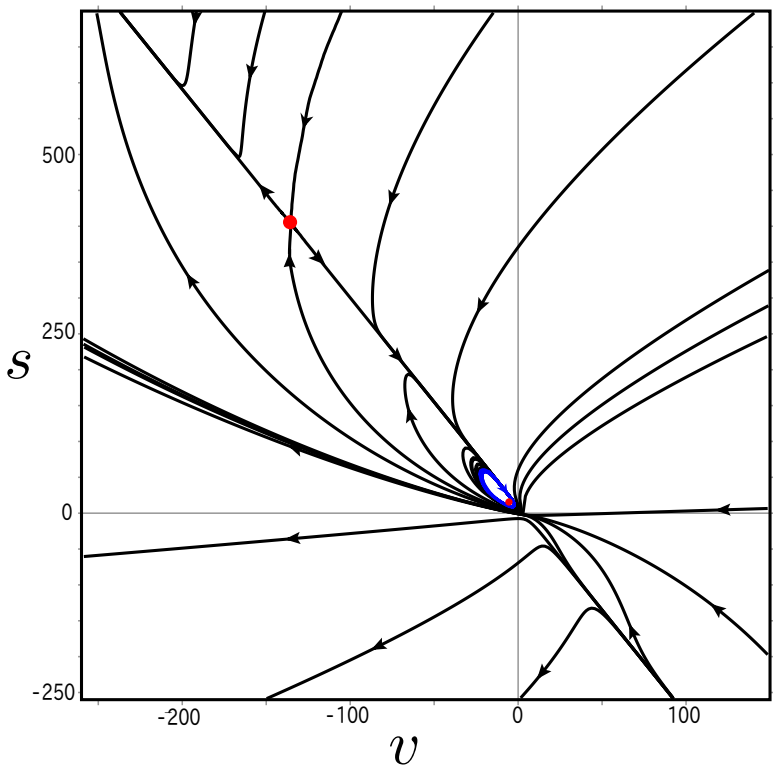

FIG. 21: The RG flows in the $v-s$ plane in a wide view. Another fixed point is shown together with the limit cycle loop.

\section{$(-135.52,403.62,-1069.65)$. See Fig. 21.}

The eigenvalues of the linearized RGEs at the fixed point located inside the limit-cycle loop are numerically obtained as $0.3288 \pm 2.3212 i$ and 1.9817 . The eigenvector of the real positive eigenvalue is in the $w$ direction. It is known that the imaginary part of the complex eigenvalues, $\theta$, gives approximately the period of the limit cycle,

$$
T \simeq \frac{2 \pi}{\operatorname{Im} \theta} .
$$

With $\theta=2.3212$, we find $T=2.7069$, which corresponds to the value of Efimov parameter $s_{0}=1.1606$.

\section{B. On the trivial fixed point}

As explained in Sec. VD, we find a nontrivial fixed point of the three-body coupling $d_{0}$ on the two-body trivial fixed point. In this section, we examine the effects of the higher order operators on it.

The RGEs on the trivial fixed point of the two-body sector are given by

$$
\begin{aligned}
\frac{d v}{d t} & =-4 v-c v^{2}-\frac{1}{3} v s-\frac{7}{120} s^{2} \\
\frac{d s}{d t} & =-6 s-c v s-\frac{1}{6} s^{2} \\
\frac{d w}{d t} & =-6 w+6 e v^{2}+\frac{1}{4} s^{2}+3 c v s-2 c v w-\frac{1}{3} s w \cdot(6.8)
\end{aligned}
$$

The RG flow in the $v$-s plane is shown in Fig. 22, We find the Borromean fixed point at the same value of $v$. In the $v-s-w$ space, the fixed point is at $\left(-4 / c, 0,-48 e / c^{2}\right) \approx$ $(-8.13,0,-74.80)$. This strongly suggests that the existence of the Borromean fixed point is not an artefact of the restriction of the set of operators. 


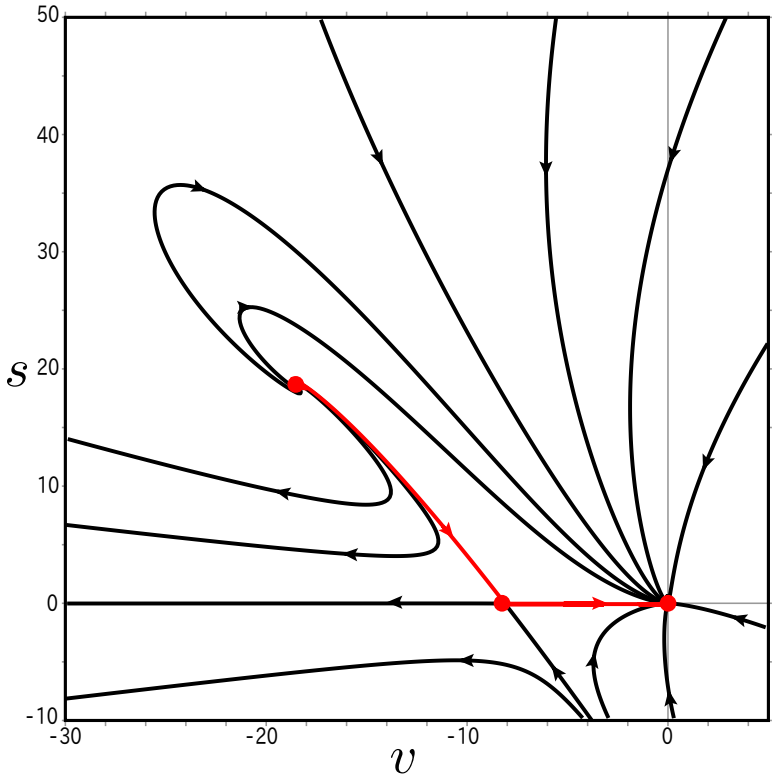

FIG. 22: The RG flow on the two-body trivial fixed point $(\xi=0)$. We find another nontrivial fixed point in addition to the trivial and Borromean fixed points.

By linearizing the RGEs at the Borromean fixed point, we find the eigenvalues, $\nu$, and the corresponding (unnormalized) eigenvectors, $(\delta v, \delta s, \delta w)$;

$$
4:\left(\begin{array}{c}
c \\
0 \\
24 e
\end{array}\right), \quad 2:\left(\begin{array}{c}
0 \\
0 \\
1
\end{array}\right), \quad-2:\left(\begin{array}{c}
2 c / 3 \\
-3 c^{2} \\
4 e-9 c^{2}
\end{array}\right) .
$$

Note that there are two relevant operators.

$\begin{array}{llrr}\text { We } \quad \text { also find other fixed points } \\ \text { at } & (-18.45,18.48,-58.81) & \text { and }\end{array}$ $(-247.09,693.75,-917.51)$. The former is located at the center of the whirl, and the latter, which is not displayed in Fig. 22 is related to the fixed point shown in Fig. 21].

Actually Fig.22 transforms into Fig.21(or Fig.201) if $\xi$ is treated as a parameter changing from zero (the trivial fixed point) to -1 (the nontrivial fixed point approached from the weak-coupling phase). See Fig. 23 to Fig. 25 The Borromean fixed point and the fixed point at the origin move together to fuse and disappear, while the whirl becomes enclosed by the limit cycle. This is an example of bifurcation.

\section{SUMMARY}

In this paper, we performed the Wilsonian RG analysis of nonrelativistic three-body systems of identical bosons without introducing the dimeron field. We explained why the multi-loop diagrams contribute to the RGEs and obtained the RGEs in the restricted space of operators. The periodic behavior in the case of infinite two-body scattering length is obtained with the value of the Efimov

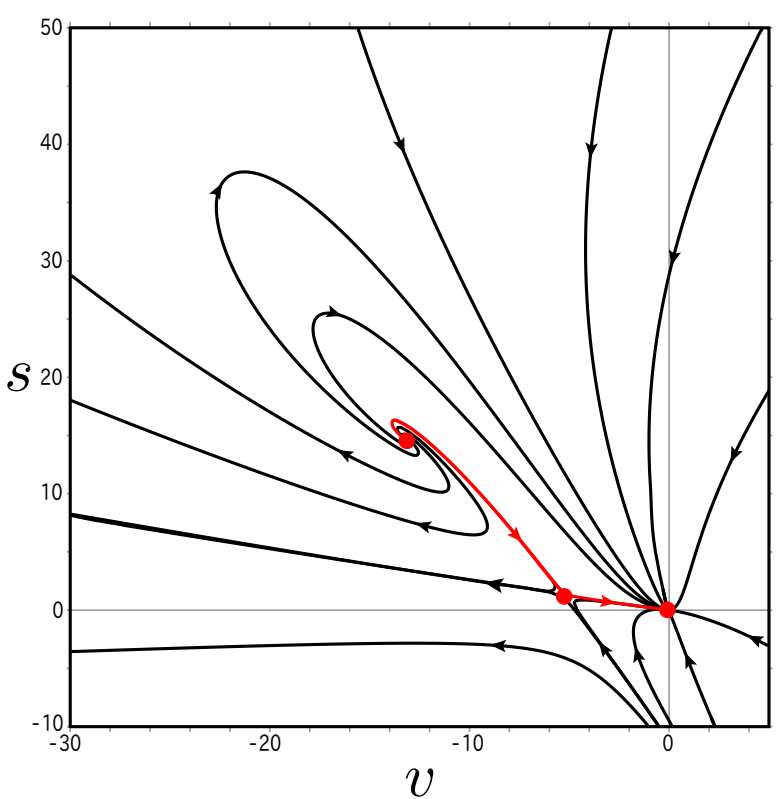

FIG. 23: The RG flow in the $v$-s plane with $\xi=-0.25$. The Borromean fixed point and the trivial fixed point move together and eventually fuse and disappear.

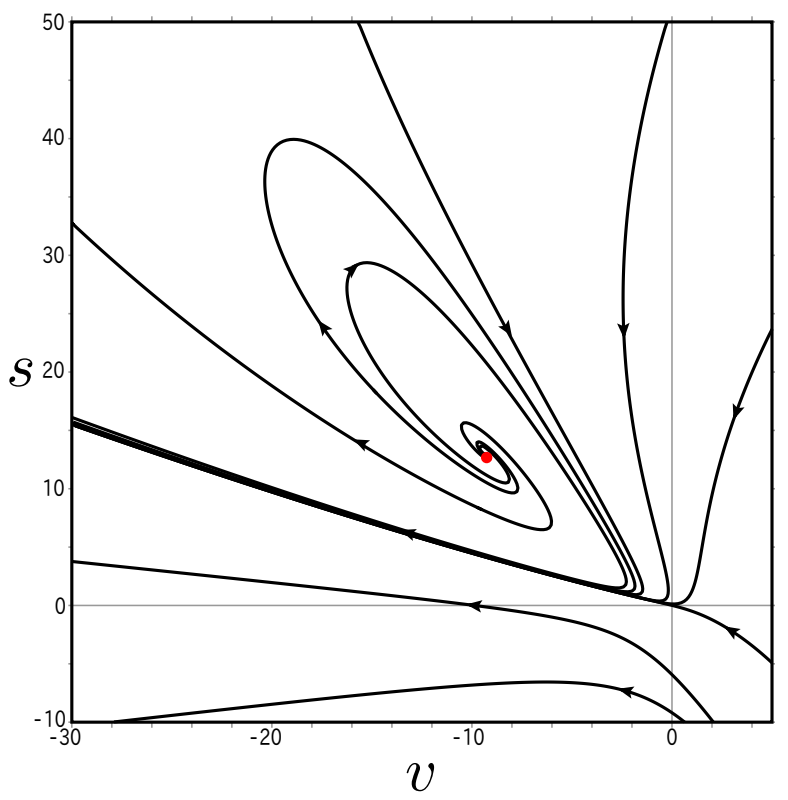

FIG. 24: The RG flow in the $v$-s plane with $\xi=-0.5$. There is no Borromean nor trivial fixed point.

parameter being very close to the Efimov's value. The difference between our result and that obtained by using the dimeron field is clarified.

We found very rich structures of the RG flows: beside the periodic behavior responsible for the Efimov effect, we found the Borromean fixed point, which, to our best knowledge, has never been noticed in the literature. It implies the existence of the three-body strong-coupling phase. We also investigated the effects of finite values of 


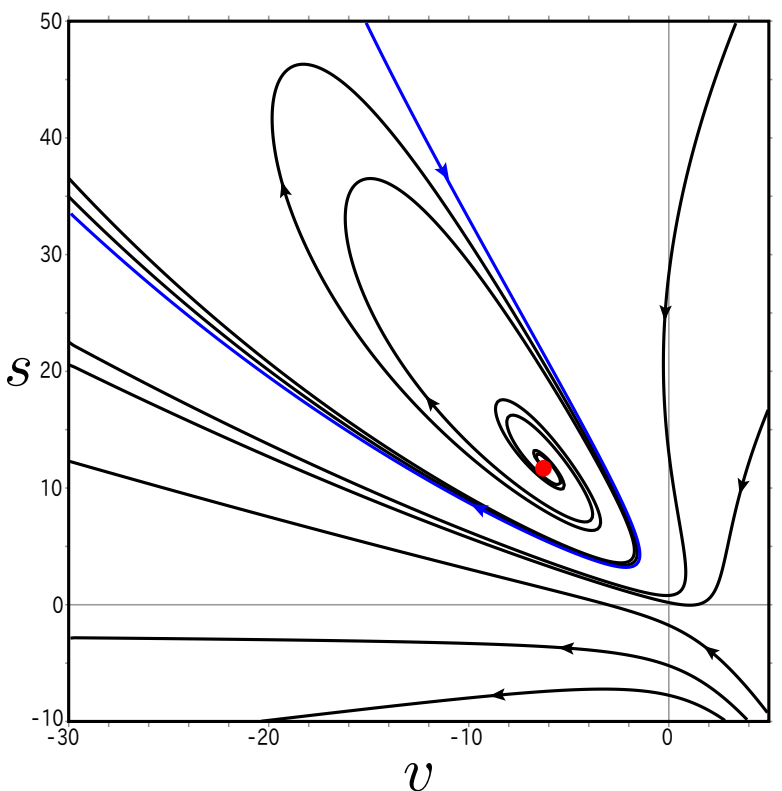

FIG. 25: The RG flow in the $v-s$ plane with $\xi=-0.75$. A large limit cycle loop emerges.

the effective range and off-shell parameters, and found that they affects the ratios of the binding energies of the three-body bound states.

We also try to explain that the binding energy of triton is larger than that of deuteron on the basis of the study of the RG flows in our bosonic system.

We then extended our set of three-body operators to $\mathcal{O}\left(p^{2}\right)$ and considered the case of the two-body fixed points. On the two-body nontrivial fixed point, the limit cycle of the three-body couplings is realized as a loop of a finite size, and two nontrivial fixed points are found. On the two-body trivial fixed point there is a nontrivial fixed point around which a whirling of the RG flow occurs, in addition to the Borromean and the trivial fixed points.

The three-body strong-coupling phase, with the phase boundary on which the Borromean fixed point resides, has a clear physical picture: it corresponds to the situation in which there are no (or very weak) two-body interactions, but a strong (short-range) attractive threebody interaction among the particles. We do not know if such a situation is possible in the real world.

\section{Acknowledgments}

I. Y. is grateful to H. Makino for his help in the early state of the investigation. The authors would like to thank J. Gegelia for the discussions and the comments on the first version of the manuscript. This work was supported by JSPS KAKENHI (Grant-in-Aid for Scientific Research (C)) (22540286).

\section{Appendix A: Use of a dimeron}

In this appendix, we illustrate a way of introducing a dimeron field in the path integral formalism, and emphasize that it is difficult to obtain the relations between the coupling constants of the original theory and those in a theory with a dimeron.

Let us start with the (Euclidean) partition function,

$$
Z=\int d \phi^{\dagger} d \phi e^{-\int d^{4} x \mathcal{L}_{E}},
$$

where $\mathcal{L}_{E}$ is the Euclidean version of the Lagrangian given by Eq. (2.1). We insert the identity,

$$
1=\int d D^{\dagger} d D e^{-\int d^{4} x \Delta D^{\dagger} D}
$$

where $\Delta$ is assumed to be a positive constant for simplicity. It can also contain derivatives. In such a case, the resulting free-part of the dimeron Lagrangian contains derivatives.

We then make a change of variables,

$$
\begin{aligned}
D & =D^{\prime}\left(1+a \phi^{\dagger} \phi+\cdots\right)+\phi^{2}\left(b+c \phi^{\dagger} \phi+\cdots\right), \quad(\mathrm{A} 3) \\
D^{\dagger} & =\left(D^{\prime}\right)^{\dagger}\left(1+a \phi^{\dagger} \phi+\cdots\right)+\left(\phi^{\dagger}\right)^{2}\left(b+c \phi^{\dagger} \phi+\cdots\right),
\end{aligned}
$$

where $a, b$, and $c$ are real constants. Here and hereafter the ellipses denote the terms of $\phi^{\prime}$ 's and $\phi^{\dagger}$ 's with derivatives. Note that we assign $D$ and $D^{\dagger}$ the particle number -2 and +2 respectively, with the assignment of the particle number -1 to $\phi$. We may include terms of higher powers in $\phi^{\dagger} \phi, D$, and $D^{\dagger}$, but such terms do not contribute to the three-body sector, and are thus ignored.

Under the change of variables, we have

$$
\begin{aligned}
\Delta D^{\dagger} D= & \Delta\left(D^{\prime}\right)^{\dagger} D^{\prime}+b \Delta\left(\left(D^{\prime}\right)^{\dagger} \phi^{2}+\text { h.c. }\right) \\
& +2 a \Delta\left(D^{\prime}\right)^{\dagger} D^{\prime} \phi^{\dagger} \phi \\
& +(c+a b) \Delta\left(\left(D^{\prime}\right)^{\dagger} \phi^{\dagger} \phi^{3}+\text { h.c. }\right) \\
& +b^{2} \Delta\left(\phi^{\dagger} \phi\right)^{2}+2 b c \Delta\left(\phi^{\dagger} \phi\right)^{3} \\
& +\cdots .
\end{aligned}
$$

This is not the whole story, however. One needs to consider the Jacobian contribution unless one uses dimensional regularization,

$$
d D^{\dagger} d D=d D^{\prime \dagger} d D^{\prime} J\left(\phi, \phi^{\dagger}\right)
$$

The Jacobian may be written as an action,

$$
J\left(\phi, \phi^{\dagger}\right)=\exp \left[-\int d^{4} x \mathcal{L}_{\text {Jacobian }}\right],
$$

up to a numerical constant, with $\mathcal{L}_{\text {Jacobian }}$ being expanded in terms of local terms,

$$
\mathcal{L}_{\text {Jacobian }}=\delta M \phi^{\dagger} \phi+A a^{2}\left(\phi^{\dagger} \phi\right)^{2}+B a^{3}\left(\phi^{\dagger} \phi\right)^{3}+\cdots,
$$


with $\delta M, A$, and $B$ are regularization dependent real constants. The calculation of the Jacobian must be consistent with the regularization of the other parts, and it is difficult to ensure the consistency. See Ref. [27] for an example of consistent calculation of a Jacobian factor in the lattice regularization. Here we do not try to calculate it, since it does not affect the following argument.

The first term in Eq. A8 may be absorbed in the definition of the mass. With the other terms being included, the total Lagrangian becomes

$$
\begin{aligned}
\mathcal{L}_{E}^{\prime}= & \mathcal{L}_{E}+\Delta\left(D^{\prime}\right)^{\dagger} D^{\prime}+b \Delta\left(\left(D^{\prime}\right)^{\dagger} \phi^{2}+\text { h.c. }\right) \\
& +2 a \Delta\left(D^{\prime}\right)^{\dagger} D^{\prime} \phi^{\dagger} \phi \\
& +(c+a b) \Delta\left(\left(D^{\prime}\right)^{\dagger} \phi^{\dagger} \phi^{3}+\text { h.c. }\right) \\
& +\left(b^{2} \Delta+A a^{2}\right)\left(\phi^{\dagger} \phi\right)^{2}+\left(2 b c \Delta+B a^{3}\right)\left(\phi^{\dagger} \phi\right)^{3} \\
& +\cdots
\end{aligned}
$$

Thus, to eliminate the terms $\left(\phi^{\dagger} \phi\right)^{2},\left(\phi^{\dagger} \phi\right)^{3}$, and $\left(D^{\prime}\right)^{\dagger} \phi^{\dagger} \phi^{3}+$ h.c. in the whole Lagrangian, we choose $a$, $b$, and $c$ to satisfy the following relations,

$$
\begin{aligned}
& 0=(c+a b) \Delta, \\
& 0=-\frac{c_{0}}{4}+b^{2} \Delta+A a^{2}, \\
& 0=-\frac{d_{0}}{36}+2 b c \Delta+B a^{3} .
\end{aligned}
$$

By eliminating $b$ and $c$ we have

$$
\left(-\frac{1}{2} B-A\right) a^{3}+\frac{c_{0}}{4} a+\frac{d_{0}}{72}=0 .
$$

There is at least one real solution for $a$. Once the solution for $a$ is obtained, the solutions for $b$ and $c$ are obtained easily. We have shown that, even in the presence of the contributions from the Jacobian, the terms $\left(\phi^{\dagger} \phi\right)^{2},\left(\phi^{\dagger} \phi\right)^{3}$, and $\left(D^{\prime}\right)^{\dagger} \phi^{\dagger} \phi^{3}+$ h.c. can be eliminated but, as we claimed before, the relations between the coupling constants of the original theory and those in the theory with the dimeron are not simple at all.

\section{Appendix B: An example of NOOL diagram contributions to the RGE for $d_{0}$}

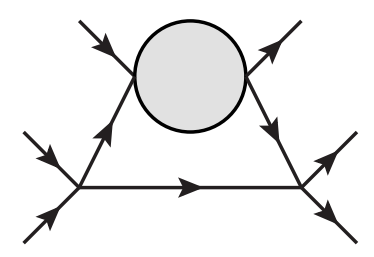

FIG. 26: An example of the NOOL diagram contributing to the RGE for $d_{0}$.

In this appendix, we illustrate how the NOOL diagram contributions are evaluated by showing an example.
The amplitude of the diagram shown in Fig. 26 is given by

$$
\begin{aligned}
& \int \frac{d^{4} k}{(2 \pi)^{4}}\left(-i c_{0}-i 4 c_{2} \boldsymbol{k}^{2}-i \frac{b_{2}}{M} \boldsymbol{k}^{2}\right)^{2} \\
& \times\left(-i \mathcal{A}\left(\mu, \frac{\boldsymbol{k}^{2}}{4}, \frac{\boldsymbol{k}^{2}}{4}\right)\right) \\
\times & {\left[\frac{i}{k^{0}-\frac{\boldsymbol{k}^{2}}{2 M}+i \epsilon}\right]^{2} \frac{i}{-k^{0}-\frac{\boldsymbol{k}^{2}}{2 M}+i \epsilon} } \\
= & -\frac{i}{2 \pi^{2}} \int_{0}^{\Lambda} d k\left(c_{0}+4 c_{2} k^{2}+\frac{b_{2}}{M} k^{2}\right)^{2} \\
& \times \mathcal{A}\left(\frac{\sqrt{3} k}{2}, \frac{k^{2}}{4}, \frac{k^{2}}{4}\right) \frac{k^{2}}{\left[-k^{2} / M+i \epsilon\right]^{2}},
\end{aligned}
$$

where the external energies and momenta are set to zero. The first factor comes from the two two-body vertices. $\mathcal{A}$ stands for the two-body amplitude (4.1), corresponding to the blob in Fig. 26. We have integrated over $k^{0}$ by picking up the pole at $k^{0}=-k^{2} / 2 M+i \epsilon$ by the contour integration on the upper half plane. Note that other poles and the cut in the amplitude is on the lower half plane. The cutoff is introduced for the relative momentum, in order to preserve the Galilean invariance of the theory 24].

The contribution to the RGE comes from the shellmode part of the integral, $\Lambda-d \Lambda<k<\Lambda$ :

$$
\begin{aligned}
& \frac{-i}{2 \pi^{2}}\left(c_{0}+4 c_{2} \Lambda^{2}+\frac{b_{2}}{M} \Lambda^{2}\right)^{2} \mathcal{A}\left(\frac{\sqrt{3} \Lambda}{2}, \frac{\Lambda^{2}}{4}, \frac{\Lambda^{2}}{4}\right) \\
& \times \frac{\Lambda^{2}}{\left[-\Lambda^{2} / M+i \epsilon\right]^{2}} d \Lambda \\
= & \frac{-i M^{2}}{2 \pi^{2} \Lambda^{2}}\left(c_{0}+4 c_{2} \Lambda^{2}+\frac{b_{2}}{M} \Lambda^{2}\right)^{2} \mathcal{A}\left(\frac{\sqrt{3} \Lambda}{2}, \frac{\Lambda^{2}}{4}, \frac{\Lambda^{2}}{4}\right) d \Lambda \\
= & -4 i \frac{2 \pi^{2}}{\Lambda^{4}}(x+y+z)^{2} \mathcal{A}\left(\frac{\sqrt{3} \Lambda}{2}, \frac{\Lambda^{2}}{4}, \frac{\Lambda^{2}}{4}\right) d \Lambda . \quad(\mathrm{B} 2)
\end{aligned}
$$

We have the same contributions from the interchanges of the external momenta, so that we multiply it with $3 \times 3$, obtaining

$$
-36 i \frac{2 \pi^{2}}{\Lambda^{4}}(x+y+z)^{2} \mathcal{A}\left(\frac{\sqrt{3} \Lambda}{2}, \frac{\Lambda^{2}}{4}, \frac{\Lambda^{2}}{4}\right) d \Lambda .
$$

The two-body amplitude in terms of $X, Y$, and $Z$ is given by

$$
\begin{aligned}
& \mathcal{A}\left(\mu, \boldsymbol{k}_{1}^{2}, \boldsymbol{k}_{2}^{2}\right) \\
= & \frac{4 \pi^{2}}{M \Lambda}\left[1-\frac{\pi}{2} \frac{\mu}{\Lambda}+\frac{X^{2}}{Y}+\frac{\mu^{2}}{\Lambda^{2}}\left(\frac{X^{2} Z}{Y^{2}}+1\right)\right. \\
& \left.-3\left(\frac{2 \mu^{2}}{\Lambda^{2}}+\frac{\boldsymbol{k}_{1}^{2}+\boldsymbol{k}_{2}^{2}}{\Lambda^{2}}\right) \frac{X(X-1)}{Y}\left(\frac{X^{2}}{Y}+1\right)\right]^{-1} .
\end{aligned}
$$


The combination $1+X^{2} / Y$ is written in terms of the two-body scattering length, $a_{2}$, as

$$
1+\frac{X^{2}}{Y}=\frac{\pi}{2} \frac{1}{a_{2} \Lambda}
$$

and the combination $X^{2} Z / Y^{2}+1$ is written in terms of the two-body effective range, $r_{e}$, as

$$
\frac{X^{2} Z}{Y^{2}}+1=\frac{\pi}{4} r_{e} \Lambda
$$

Note that the cutoff $\Lambda$ is small enough so that the effective range expansion is valid. If we denote the two-body cutoff $\Lambda_{0}^{(2)}$, above which the effective theory description breaks down, we assume

$$
\Lambda \ll \Lambda_{0}^{(2)}
$$

We also assume that the effective range is of a natural size, i.e.,

$$
r_{e} \Lambda_{0}^{(2)} \sim \mathcal{O}(1)
$$

while the scattering length may (or may not) be finetuned. Thus, we have

$$
r_{e} \Lambda \ll 1 .
$$

The combination $3 X(X-1)\left(X^{2} / Y+1\right) / Y$ is related to the off-shell parameter $h$ through

$$
\frac{X(X-1)}{Y}\left(\frac{X^{2}}{Y}+1\right)=\frac{\pi}{2} \frac{h \Lambda^{2}}{a_{2} \Lambda} .
$$

We assume that $h$ is also of a natural size,

$$
h\left(\Lambda_{0}^{(2)}\right)^{2} \sim \mathcal{O}(1)
$$

thus

$$
h \Lambda^{2} \ll 1
$$

Therefore the quantity in Eq. (B10) is much smaller than the one in Eq. (B5).

We may expand the corresponding terms in $\mathcal{A}\left(\sqrt{3} \Lambda / 2, \Lambda^{2} / 4, \Lambda^{2} / 4\right)$, keeping $1+X^{2} / Y$ in the denominator. We finally get

$$
-72 i \frac{\left(2 \pi^{2}\right)^{2}}{M \Lambda^{5}} \frac{V^{2}}{S^{2}}(T-6 U) d \Lambda,
$$

where $S, T, U$, and $V$ are defined in Eqs. (4.16).

\section{Appendix C: Shell mode contribution of the two-loop diagram with three symmetric lines}

In this Appendix, we consider the shell mode contribution of the two-loop diagram shown in the fifth diagram in Fig. 12

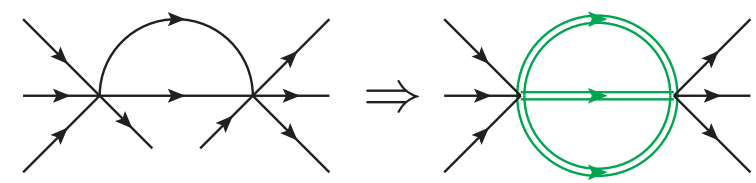

FIG. 27: The NOOL diagram, in which the identification of the shell mode contribution is not so obvious.

This NOOL diagram is obtained by contracting two legs of the one-loop diagram as shown in Fig. 27. The additional line connects the same vertices as two lines in the original diagram do. As we explained in Sec. IIIB. the three lines should be treated in a symmetric way and we should impose the cutoff so that Galilean invariance is maintained. By doing so, the momenta of the three lines are related to the shell mode.

A trouble is that there seems no obvious way to do so by just "modifying" the shell mode momentum assignment, as we did in Fig. 4. Fortunately, however, there is a trivial way to identify the shell mode contribution of the diagram starting from the amplitude,

$$
\begin{aligned}
I(\Lambda) \equiv & \frac{1}{6}\left(-i d_{0}\right)^{2} \int \frac{d^{4} k}{(2 \pi)^{4}} \int \frac{d^{4} l}{(2 \pi)^{4}} \frac{i}{-k^{0}-\boldsymbol{k}^{2} / 2 M+i \epsilon} \\
& \times \frac{i}{k^{0} / 2+l^{0}-(\boldsymbol{k} / 2+\boldsymbol{l})^{2} / 2 M+i \epsilon} \\
& \times \frac{i}{k^{0} / 2-l^{0}-(\boldsymbol{k} / 2-\boldsymbol{l})^{2} / 2 M+i \epsilon}, \quad(\mathrm{C} 1)
\end{aligned}
$$

where the external energy and momentum are set to zero for simplicity. The domain of the momentum integrations is restricted to the region $|\boldsymbol{k}|<\Lambda$ and $|\boldsymbol{l}|<\Lambda$. These cutoffs do not break the Galilean invariance. Thus, the shell mode contribution is identified as $I(\Lambda)-I(\Lambda-d \Lambda)$. A simple calculation leads to

$$
I(\Lambda)-I(\Lambda-d \Lambda)=6 \frac{i\left(2 \pi^{2}\right)^{2}}{M \Lambda^{5}} c v^{2} d \Lambda .
$$

\section{Appendix D: Pole at $\xi=1 / a$}

In this appendix, we demonstrate that the pole at $\xi=$ 1/a appearing in Eq. (5.2) is due to the existence of a two-body bound state.

In Appendix B, we have shown how the two-body amplitude is embedded in the three-body NOOL diagrams and contributes to the RGE for $v$. The two-body amplitude in the shell mode is given by

$$
\mathcal{A}\left(\frac{\sqrt{3} \Lambda}{2}, \frac{\Lambda^{2}}{4}, \frac{\Lambda^{2}}{4}\right),
$$

and we have argued that the effective range and the offshell parameter terms can be expanded, so that effectively the amplitude appears as

$$
\mathcal{A} \sim \frac{8 \pi}{M \Lambda}\left[\frac{1}{a_{2} \Lambda}-\frac{\mu}{\Lambda}\right]^{-1},
$$




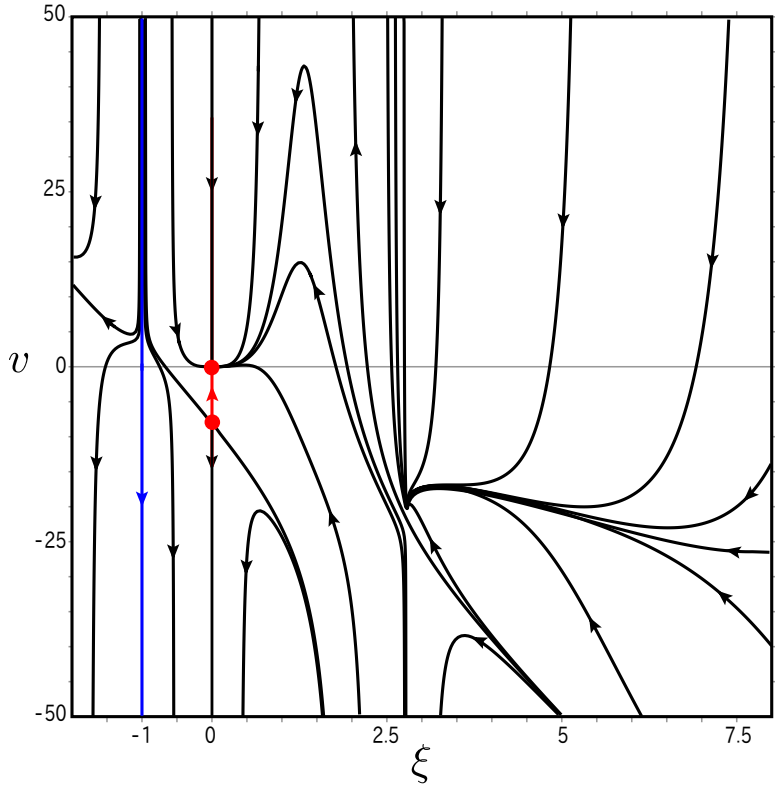

FIG. 28: The RG flow in a wider region of the $\xi-v$ plane. A "wall" is seen at $\xi=1 / a$. Rapid change of $v$ near the "wall" is to cancel the enhancement due to the factor $1 /(1-a \xi)$ in the RGE (5.2).

with $\mu=\sqrt{3} \Lambda / 2$. In the usual off-shell amplitude, $\mu$ is written as

$$
\mu=\sqrt{-M P^{0}+\boldsymbol{P}^{2} / 4-i \epsilon},
$$

the value of $\mu$ corresponds to the energy

$$
P^{0}=-\left(\frac{\sqrt{3}}{2}\right)^{2} \frac{\Lambda^{2}}{M},
$$

when the total momentum $\boldsymbol{P}$ is zero. On the other hand, because of Eq. (B5),

$$
1+\frac{1}{\xi}=\frac{\pi}{2} \frac{1}{a_{2} \Lambda},
$$

$\xi=1 / a$ corresponds to

$$
\frac{1}{a_{2} \Lambda}=\frac{\sqrt{3}}{2} .
$$

Substituting it to Eq. (D4), we get

$$
P^{0}=-\frac{1}{M a_{2}^{2}},
$$

which is nothing but the energy of a two-body bound state in the present approximation.

In the two-body RGEs, no value of the cutoff hits the pole of the amplitude, since $\mu$ is pure imaginary for any physical energy-momentum. In the present case, the twobody amplitude is embedded in the three-body diagrams, thus the "total energy-momentum" of the two-body amplitude can take any real values so that $\mu$ can be real and hits the pole of the amplitude Eq. (D2) as the value $a_{2} \Lambda$ changes. When $\xi$ comes close to $1 / a$, the three-body shell-mode contributions become huge, and the value of $v$ changes rapidly to cancel the cutoff dependence. Near the $\xi=1 / a$, the flows thus run almost vertically as shown in Fig. 28

There is however a "gate" , $(1 / a,-2 / a b)$, at which the right hand side of Eq. (5.2) is finite when $\xi$ approaches $1 / a$. Apparently no flow can pass the $\xi=1 / a$ "wall" without getting through the "gate."
[1] N. Kalantar-Nayestanaki, E. Epelbaum, J. Messchendorp, and A. Nogga, Rept.Prog.Phys. 75, 016301 (2012), 1108.1227

[2] M. Zhukov, B. Danilin, D. Fedorov, J. Bang, I. Thompson, et al., Phys.Rept. 231, 151 (1993).

[3] V. Efimov, Phys.Lett. B33, 563 (1970).

[4] V. Efimov, Sov.J.Nucl.Phys. 12, 589 (1971).

[5] P. F. Bedaque, H. W. Hammer, and U. van Kolck, Nucl. Phys. A646, 444 (1999), nucl-th/9811046.

[6] E. Braaten and H. W. Hammer, Phys. Rept. 428, 259 (2006), cond-mat/0410417.

[7] H.-W. Hammer and L. Platter, Phil.Trans.Roy.Soc.Lond. A369, 2679 (2011), 1102.3789.

[8] D. B. Kaplan, Nucl. Phys. B494, 471 (1997), nuclth/9610052.

[9] V. Efimov, Phys.Rev. C44, 2303 (1991).

[10] V. Efimov, Phys.Rev. C47, 1876 (1993).

[11] S. Moroz, S. Floerchinger, R. Schmidt, and C. Wetterich, Phys.Rev. A79, 042705 (2009), 0812.0528.

[12] S. Diehl, H. Krahl, and M. Scherer, Phys.Rev. C78, 034001 (2008), 0712.2846.
[13] B. Krippa, N. R. Walet, and M. C. Birse, Phys.Rev. A81, 043628 (2010), 0911.4608.

[14] S. Weinberg, Physica A96, 327 (1979), festschrift honoring Julian Schwinger on his 60th birthday.

[15] K. Wilson and J. B. Kogut, Phys.Rept. 12, 75 (1974).

[16] S. Weinberg, Phys. Lett. B251, 288 (1990).

[17] S. Weinberg, Nucl. Phys. B363, 3 (1991).

[18] E. Epelbaum, H.-W. Hammer, and U.-G. Meissner, Rev. Mod. Phys. 81, 1773 (2009), 0811.1338.

[19] F. J. Wegner and A. Houghton, Phys. Rev. A8, 401 (1973).

[20] J. Polchinski, Nucl. Phys. B231, 269 (1984).

[21] C. Wetterich, Phys. Lett. B301, 90 (1993).

[22] T. R. Morris, Int. J. Mod. Phys. A9, 2411 (1994), hep$\mathrm{ph} / 9308265$.

[23] M. C. Birse, J. A. McGovern, and K. G. Richardson, Phys. Lett. B464, 169 (1999), hep-ph/9807302.

[24] K. Harada and H. Kubo, Nucl. Phys. B758, 304 (2006), nucl-th/0605004.

[25] K. Harada, H. Kubo, and Y. Yamamoto, Phys.Rev. C83, 034002 (2011), 1012.2716. 
[26] K. Harada, K. Inoue, and H. Kubo, Phys. Lett. B636, 305 (2006), nucl-th/0511020.

[27] K. Harada, N. Hattori, H. Kubo, and Y. Yamamoto,
Phys. Rev. D79, 065037 (2009), 0902.0665. 